\title{
Rise and fall of molecular clouds across the M 33 disk
}

\author{
Edvige Corbelli ${ }^{1}$, Jonathan Braine ${ }^{2}$, and Carlo Giovanardi ${ }^{1}$ \\ 1 INAF-Osservatorio Astrofisico di Arcetri, Largo E. Fermi, 5, 50125 Firenze, Italy \\ e-mail: edvige@arcetri.astro.it \\ 2 Laboratoire d'Astrophysique de Bordeaux, Univ. Bordeaux, CNRS, B18N, Allée Geoffroy Saint-Hilaire, 33615 Pessac, France \\ Received 16 October 2018 / Accepted 4 January 2019
}

\begin{abstract}
We carried out deep searches for CO line emission in the outer disk of M33, at $R>7 \mathrm{kpc}$, and examined the dynamical conditions that can explain variations in the mass distribution of the molecular cloud throughout the disk of M33. We used the IRAM-30 m telescope to search for CO lines in the outer disk toward 12 faint mid-infrared (MIR) selected sources and in an area of the southern outer disk hosting MA1, a bright HII region. We detect narrow CO lines at the location of two MIR sources at galactocentric distances of about $8 \mathrm{kpc}$ that are associated with low-mass young stellar clusters, and at four locations in the proximity of MA1. The paucity of $\mathrm{CO}$ lines at the location of weak MIR-selected sources probably arises because most of them are not star-forming sites in M 33, but background sources. Although very uncertain, the total molecular mass of the detected clouds around MA1 is lower than expected given the stellar mass of the cluster, because dispersal of the molecular gas is taking place as the HII region expands. The mean mass of the giant molecular clouds (GMCs) in M 33 decreases radially by a factor 2 from the center out to $4 \mathrm{kpc}$, then it stays constant until it drops at $R>7 \mathrm{kpc}$. We suggest that GMCs become more massive toward the center because of the fast rotation of the disk, which drives mass growth by coalescence of smaller condensations as they cross the arms. The analysis of both $\mathrm{HI}$ and $\mathrm{CO}$ spectral data gives the consistent result that corotation of the two main arms in this galaxy is at a radius of $4.7 \pm 0.3 \mathrm{kpc}$, and spiral shock waves become subsonic beyond $3.9 \mathrm{kpc}$. Perturbations are quenched beyond $6.5 \mathrm{kpc}$, where CO lines have been detected only around sporadic condensations associated with UV and MIR emission.
\end{abstract}

Key words. ISM: clouds - Galaxy: kinematics and dynamics - galaxies: ISM - galaxies: individual: M 33 - ISM: molecules

\section{Introduction}

Our knowledge of molecular clouds and the processes in the interstellar medium (ISM) that favor the birth of stars is mostly based on Galactic studies. The increase in resolution and sensitivity has enabled recent extragalactic surveys to study the formation of stars as galaxies evolve and as a function of galaxy mass, morphology, and metallicity. Nearby galaxies are an ideal place where a global picture of the disk is complemented by detailed observation of the ISM and star-forming (hereafter SF) sites. In this context, particular attention has been given to low-luminosity Local Group galaxies with subsolar metallicity: M33 in the north and the Large Magellanic Cloud (LMC) in the south. The spiral galaxy M33 is particularly interesting because it is a blue SF galaxy with no evident sign of ongoing or past interactions, as shown by the most recent proper motion measurements and past orbital history (Patel et al. 2017; van der Marel et al. 2019). It is a relatively unperturbed spiral with no bulge (Corbelli \& Walterbos 2007) and an extended warped outer disk (Corbelli et al. 2014). This makes it an ideal laboratory to study how the gas settles in the disk and develops instabilities that condense and form stars and/or to determine the role of feedback in triggering a new generation of stars (Dobbs et al. 2018; Corbelli et al. 2018).

The investigation of individual SF complexes in nearby galaxies and all-disk surveys at infrared and millimeter wavelengths (through facilities such as Spitzer, Herschel, IRAM, and ALMA) reveals how the ISM and the star formation process differ in chemically young environments as compared to more evolved spirals. The molecular cloud mass spectrum steepens, the conversion of molecular gas into stars is faster, and the CO-to- $\mathrm{H}_{2}$ ratio decreases in lower metallicity environments (Gratier et al. 2012, 2010; Rosolowsky 2005; Fukui et al. 2008; Verley et al. 2009; Gil de Paz et al. 2007; Dib et al. 2011). M 33 is an ideal object to study these differences because its metallicity is only a factor two below solar (Magrini et al. 2010) and it retains a clear disk spiral morphology such that morphological and chemical differences are not mixed. Because of the limited differences, we can continue to use the same tracers as in large spirals. M 33 represents a sort of stepping stone toward smaller objects where chemical differences are more extreme and mixed with morphological changes.

Recently, Corbelli et al. (2017) have estimated the duration of the life cycle of giant molecular clouds (hereafter GMCs) in M33 using the all-disk CO $J=2-1$ survey (Druard et al. 2014) to identify 566 GMCs in the SF disk, and a mid-infrared (hereafter MIR) selected sample of 630 young stellar cluster candidates (hereafter YSCCs) from the catalog of Sharma et al. (2011). The GMCs spend 4 Myr in the inactive phase and $10 \mathrm{Myr}$ in the active SF phase. Sources are in the embedded phase (detected only in the infrared) during only $2 \mathrm{Myr}$ of the active phase, and for the remaining time YSCCs break through the cloud and become also detectable in $\mathrm{H} \alpha$ or UV. The correlation between GMCs and MIR-selected YSCCs is remarkable: in the active phase, all GMCs are associated with MIR sources.

There is, however, a non-negligible fraction of MIR sources that are not hosted by GMCs, and this fraction increases moving radially outward. One possibility is that these MIR sources are associated with less massive molecular clouds below the detection limit, which form low-mass clusters that are often 
undetectable in $\mathrm{H} \alpha$ because of the lack of massive stars. Through the MIR source catalog and $\mathrm{CO}$ pointed observations, Corbelli et al. (2011) have found low-mass SF complexes associated with molecular clouds that are weak in CO $J=$ $1-0$ and $J=2-1$ line emission. A key question related to this issue is to understand variations in the molecular mass spectrum beyond galactocentric radii of about $4 \mathrm{kpc}$ in M33 (Braine et al. 2018). We know that the mass spectrum of molecular clouds changes across the disk of a galaxy (Rosolowsky 2005; Gratier et al. 2012; Heyer \& Dame 2015), but the reason for this, and whether other properties change, is still an open question (Bigiel et al. 2010; Colombo et al. 2014; Freeman et al. 2017; Kobayashi et al. 2017). The onset of instabilities and the ability of the gas to cool and fragment may be not efficient enough to create massive complexes at large galactocentric radii, or it might just be that the spiral pattern cannot accumulate gas and merge clouds to make more massive complexes beyond corotation, although disk instabilities can still trigger the formation of filaments (Elmegreen \& Elmegreen 1995; Dobbs et al. 2018).

Outer disks are key places to understand galaxy evolution: in the local Universe, $\Lambda \mathrm{CDM}$ models predict that large spirals like M31 do not accrete much gas into the disk, while lowmass galaxies, such as M33, experience an inside-out growth and feed star formation through cold gas streams from the intergalactic medium that settle into the disk (Magrini et al. 2007; Williams et al. 2009; Kereš et al. 2009; Dekel et al. 2013; Fitts et al. 2018). It is therefore important to understand how pristine outer gas mixes with the galaxy ISM, and in particular, if and how star formation and metal enrichment occur beyond the bright SF disk and if this occurs continuously or in a burst. Imaging in $\mathrm{V}$ and I band with the Subaru/Suprime-Cam of a few fields of M33 in the outer regions (Grossi et al. 2011) has revealed a pervasive diffuse evolved stellar population (>1 Gyr), but also a population of younger stars (100-200 Myr) where HI gas overdensities are present in the outer disk. An interesting output of MIR source selection in the M33 area is that lowluminosity sources are also present in correspondence with the outer disk beyond the SF edge where the $\mathrm{H} \alpha$ surface brightness drops (Verley et al. 2009; Sharma et al. 2011). One possibility is that a background population of faint MIR sources is mixed in projection with truly M33 SF sites and that this becomes the dominant population at large radii. It may also be, however, that some of these faint MIR sources are small SF sites in the outer disk of M33. In this paper we use sensitive IRAM-30 m observations to understand the nature of these faint MIR sources. It is conceivable to detect $\mathrm{CO}$ emission in molecular clouds just beyond the SF edge in M 33 because the metallicity is about onethird solar (Grossi et al. 2011; Magrini et al. 2010) and the UV field is weak (Thilker et al. 2005). It is still unclear which are the conditions that favor the growth of molecular clouds beyond the SF edge of a galaxy, and whether it is possible to find them in the extreme outer disk (Digel et al. 1994; Snell et al. 2002; Braine et al. 2007).

In Sect. 2 we summarize some properties of GMCs and MIR sources across the M 33 disk. In Sect. 3 we describe CO observations in the outer disk for a selected MIR sample and around a bright HII region. In Sect. 4 we discuss some implications of the detected and undetected CO lines, such as the molecular mass of condensations in the outer disk and the nature of faint MIR sources. In Sect. 5 we show results relative to disk dynamics that can be linked to the observed radial distribution of molecular clouds. Section 6 concludes after we summarize the main results.

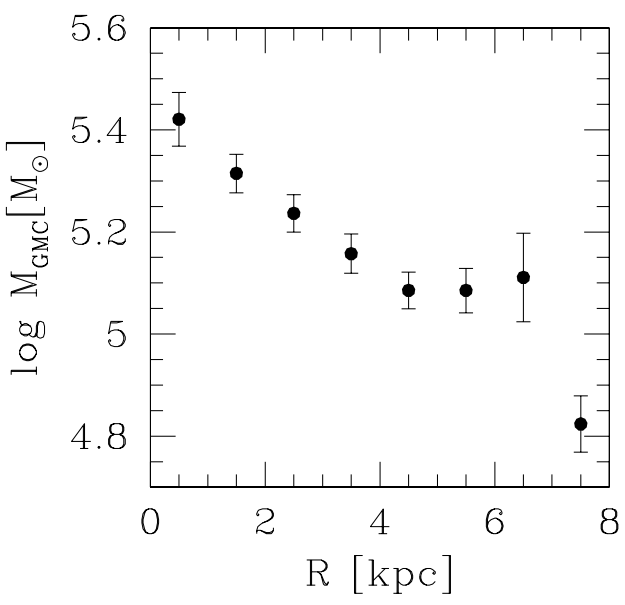

Fig. 1. Mean value of GMC mass in dex in radial bins of galactocentric distance from data in the GMC catalog of Corbelli et al. (2017).

\section{Distribution of GMCs and MIR sources across the disk of M 33}

M33 has a gaseous disk with holes and dense filaments related to optically visible flocculent spiral arms. Two of these have more prominent and numerous HII regions, one is in the northern approaching side and the other is in the southern receding side. We examine three distinct radial ranges, $R<4 \mathrm{kpc}$, $4<R<7 \mathrm{kpc}$, and $R \geq 7 \mathrm{kpc}$, and we refer to these as inner disk (dominated by the two main arms), the intermediate disk, and the outer disk. In this paper we refer to the SF disk of M33 to indicate the area within galactocentric distances of $7 \mathrm{kpc}$ (inner + intermediate disk). The outer disk encloses the extreme regions of the optical disk close to $R_{25}$ and the warp, and it is beyond the $\mathrm{H} \alpha$ luminosity drop (Kennicutt 1989; Verley et al. 2009). In this section we review the changes in the mean mass of GMCs and the $24 \mu \mathrm{m}$ flux of MIR sources across the M 33 disk. We also describe the selection of the MIR sample for deep $\mathrm{CO}$ observations in the outer disk.

The GMC catalog comprises 566 clouds with masses between $2 \times 10^{4}$ and $2 \times 10^{6} M_{\odot}$ and radii between 10 and $100 \mathrm{pc}$ (Corbelli et al. 2017). We recall that for the existing GMC cata$\log$ and in the rest of this paper, the CO-to- $\mathrm{H}_{2}$ conversion factor used is constant and equal to $4 \times 10^{20} \mathrm{~K}^{-1} \mathrm{~km}^{-1} \mathrm{~s} \mathrm{~cm}^{-2}$ unless stated otherwise. The estimated mass of 490 clouds is above the survey completeness limit of $6.3 \times 10^{4} M_{\odot}$. Braine et al. (2018) have shown that the GMC mass spectrum becomes steeper moving towards large galactocentric radii, and the maximum mass of GMCs is also a factor 2 smaller than in the inner disk. A similar trend has been found for the $24 \mu \mathrm{m}$ flux of the MIR source in the M 33 area by Sharma et al. (2011): the cumulative distribution steepens in the intermediate disk, where the maximum source luminosity is lower than in the inner disk. The ratio of molecular cloud mass to MIR flux density at $24 \mu \mathrm{m}$ does not show a clear radial trend: it has a mean value of about $4 \times 10^{4} M_{\odot} \mathrm{mJy}^{-1}$ and a large dispersion (of about $0.7 \mathrm{dex}$ ) at all radii.

The number of GMCs per unit area decreases continuously with radius across the disk. However, the average mass of GMCs decreases radially in the inner disk (which hosts 410 GMCs), but then it flattens in the intermediate disk (hosting 152 GMCs) and drops in the outer disk (where only 4 GMCs are found since the all-disk survey does not cover much of the outer disk). Figure 1 shows this radial trend. The analysis of Gratier et al. (2017), dedicated to investigating whether and how CO traces 


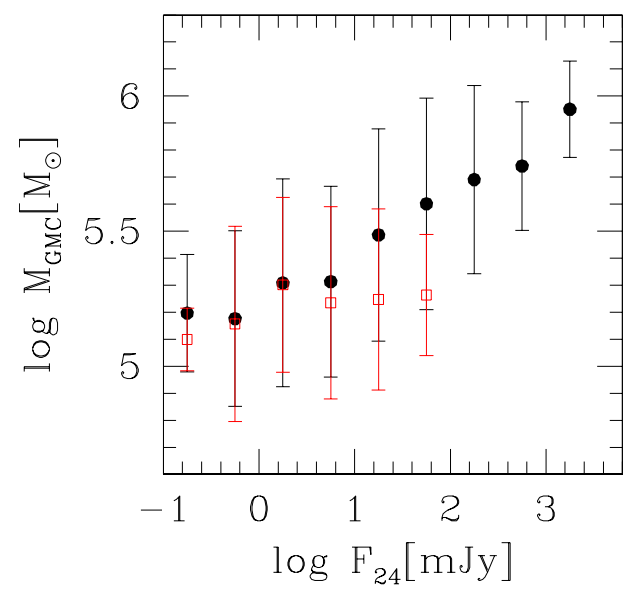

Fig. 2. Mean value of GMC mass hosting MIR sources in the SF disk of M 33 (black filled circles) in dex and in bins of $F_{24}$, the $24 \mu \mathrm{m}$ source flux. Red open squares refer to GMCs and MIR sources in the intermediate disk.

molecular cloud masses in the SF disk, confirms the rather constant fraction of dark gas across the SF disk that is, that the CO-to- $\mathrm{H}_{2}$ conversion factor does not increase at large galactocentric radii in the SF disk. This ensures that the observed trend for the mean molecular mass in the inner and intermediate disk, inferred for a constant $\mathrm{CO}-$ to- $\mathrm{H}_{2}$ conversion facto, is indeed real. The increase in mean GMC mass in the inner disk can be caused by the rotation of the spiral arm pattern, which collects clouds into more massive complexes inside $4 \mathrm{kpc}$. This mechanism can break beyond a certain radius, the corotation radius. Farther out, beyond $6.5 \mathrm{kpc}$, the disk is stable according to the Toomre stability criterion (Corbelli 2003). Alternatively, the shear may break the perturbations apart at large radii and leave only GMCs below a certain mass. We examine these possibilities in Sect. 5 after discussing the results of additional $\mathrm{CO}$ observations of selected targets in the outer disk in the next sections.

The mean mass of molecular clouds hosting MIR sources is weakly related to the intensity of the $24 \mu \mathrm{m}$ flux, $F_{24}$, for bright sources. This is shown in Fig. 2, where we compute the mean GMC mass in bins of $24 \mu \mathrm{m}$ flux intensity. For $F_{24}>5 \mathrm{mJy}$, the mean GMC mass increases as the $24 \mu \mathrm{m}$ flux of the hosted source increases. However, this trend has a large scatter, and furthermore, it disappears for faint MIR sources or if we consider only MIR sources in the intermediate disk, which is devoid of bright MIR sources (open red squares in Fig. 2). This can be due to the limited sensitivity and completeness of the $\mathrm{CO}$ all-disk survey, and it needs further investigation by deeper $\mathrm{CO}$ searches around faint MIR sources. However, when we extrapolate the trend observed for $F_{24}>5 \mathrm{mJy}$, the expected mean mass of a GMC hosting an MIR source with $F_{24} \simeq 0.1-1 \mathrm{mJy}$ is about $10^{5} M_{\odot}$, only one order of magnitude below the mean mass of GMCs that are associated with the brightest MIR sources $\left(F_{24} \sim 10^{3} \mathrm{mJy}\right)$. It is then conceivable to detect molecular gas associated with faint MIR sources through pointed observations, even though the $\mathrm{CO}-$ to- $\mathrm{H}_{2}$ ratio might decrease in the outer disk. Molecular line emission has been detected in the SF disk of M 33 by Corbelli et al. (2011) at the location of compact MIR sources with $3 \leq F_{24} \leq 21 \mathrm{mJy}$ at the level of $0.3 \mathrm{~K} \mathrm{~km} \mathrm{~s}^{-1}$. Estimated molecular cloud masses range between $10^{4}$ and $10^{5} M_{\odot}$. The detected lines suggest that low-mass GMCs might be ubiquitous around MIR sources in the SF disk of M33, despite the large spread in CO-to- $F_{24}$ flux ratio. This needs to be examined for fainter MIR sources and in the outer disk.
In the rest of this section, we select a faint MIR sample in the outer disk for deep pointed $\mathrm{CO}$ observations. These observations are presented in the next section, where we additionally describe the results of a CO $J=2-1$ line map around a bright $\mathrm{H} \alpha$ source in the outer disk.

\subsection{Multiwavelength data}

In analyzing the data of M 33, we assumed a distance of $840 \mathrm{kpc}$ (Freedman et al. 1991; Gieren et al. 2013), which implies a linear scale of $4.1 \mathrm{pc}$ per arcsecond.

Dust emission at MIR wavelengths has been investigated through the InfraRed Array Camera (IRAC) and the Multiband Imaging Photometer (MIPS) on board Spitzer. The complete set of IRAC $(3.6,4.5,5.8$, and $8.0 \mu \mathrm{m})$ and MIPS (24, and $70 \mu \mathrm{m}$ ) images of M 33 is described by Verley et al. (2007). To investigate the ultraviolet (UV) continuum emission of M33, we used Galaxy Evolution Explorer (GALEX) data, in particular those distributed by Gil de Paz et al. (2007). To trace ionized gas, we adopted the narrow-line $\mathrm{H} \alpha$ image of M 33 obtained by Greenawalt (1998) and described in detail in Hoopes \& Walterbos (2000).

The H $\alpha$ and FUV surface brightnesses in M 33 decrease radially with a scale length of about $2 \mathrm{kpc}$ out to about $6.5 \mathrm{kpc}$ (Verley et al. 2009). Beyond this radius, they experience a sharper radial decline (see also Kennicutt 1989). The distribution of the neutral atomic gas traced by $21 \mathrm{~cm}$ line emission in M 33 has been mapped by combining Very Large Array (VLA) and the Green Bank Telescope (GBT), and results are described by Corbelli et al. (2014). The atomic gas distribution starts to warp beyond the SF disk, and the surface mass density drops at $R_{25}$ (which is about $8.6 \mathrm{kpc}$ ), where the warp becomes severe. We used the tilted ring model fitted to the $21 \mathrm{~cm}$ velocity field (Corbelli et al. 2014) to estimate galactocentric distances for sources in the warped outer disk.

\subsection{Selection of the MIR sample in the outer disk}

From the catalog of 912 MIR sources in the M33 area (Sharma et al. 2011), we selected 99 sources that might be associated with SF sites at galactocentric distances $7.5<R<$ $10.8 \mathrm{kpc}$ and that have not been listed as M 33 variable stars or have a Milky Way star as optical counterpart. The inner radial cutoff was chosen to avoid overlaps with the M33 all-disk CO $J=2-1$ survey covering the SF disk (Druard et al. 2014), while the outer radius was dictated by the full coverage of SpitzerIRAC maps of M 33 and of other available maps (GALEX, $\mathrm{H} \alpha$ ). The analysis of $\mathrm{CO}$ observations of a previous sample of faint, compact MIR sources in the SF disk of M 33 has revealed the importance of IRAC and MIR color-color diagram as a diagnostic tool to distinguish MIR sources that are truly SF sites from evolved stellar objects, such as AGB stars and background galaxies (Corbelli et al. 2011). In the SF disk, CO $J=1-0$ and 2-1 lines have been detected around all MIR sources with a characteristic spectral energy distribution between 3.6 and $24 \mu \mathrm{m}$ (see their Fig. 5). The main-beam integrated temperature for the $\mathrm{CO}$ $J=2-1$ lines for the selected MIR sources in the SF disk is between 0.7 and $2 \mathrm{~K} \mathrm{~km} \mathrm{~s}^{-1}$ with peaks between 0.1 and $0.5 \mathrm{~K}$ (Corbelli et al. 2011) that are uncorrelated with the intensity of $24 \mu \mathrm{m}$ flux (in the range 1-21 $\mathrm{mJy}$ ).

To estimate the energy density in IRAC bands, we performed aperture photometry for the 99 selected sources with a fixed aperture size of 8 arcsec. We refer to Sharma et al. (2011) for details on the photometry at $24 \mu \mathrm{m}$, in $\mathrm{H} \alpha$, FUV, and NUV bands 


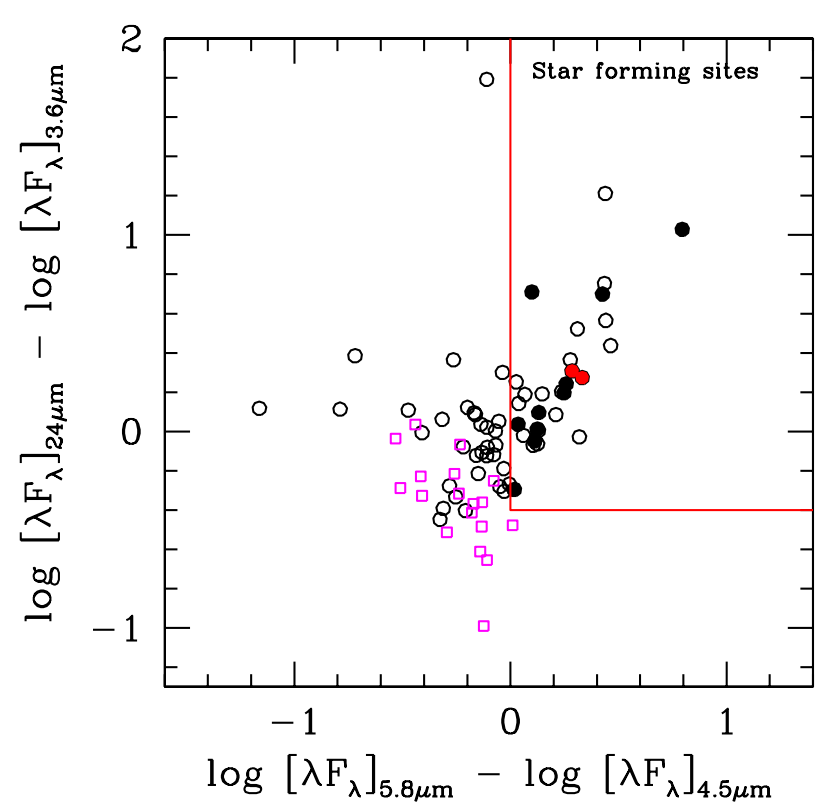

Fig. 3. Colors or differences in energy densities at two wavelengths for MIR sources in the catalog of Sharma et al. (2011) that are at galactocentric distances between 7.5 and $10.8 \mathrm{kpc}$. The red box selects $30 \mathrm{MIR}$ sources that are likely to be YSCCs. The filled symbols indicate the YSCCs selected for pilot observations of the CO $J=1-0$ and $J=2-1$ lines presented in this paper. Filled red symbols show the two YSCCs where the $\mathrm{CO}$ lines have been detected. The open magenta squares indicate the location of MIR sources that optically appear as background disk galaxies.

and for the relative uncertainties. In the red box in the upper right corner of Fig. 3 we show the 30 MIR sources that have [5.8]-[4.5] $>0$ and [24]-[3.6] $>-0.4$ flux ratios and are our best YSCCs beyond the edge of the SF disk of M33. Using the optical Sloan Digital Sky Survey images, we tagged 18 of the 99 sources that have an optical counterpart that is shaped as a disk galaxy. The 18 tagged sources, which are likely background disk galaxies, are plotted using open magenta squares. Figure 3 shows that none of them lie in the selected region (red box). However, we cannot completely exclude contamination from background galaxies, which are unresolved by the SDSS survey or are not disk like, and we discuss this possibility further in Sect. 3. For the pilot observations presented here, we selected 12 of the 30 YSCCs as a representative sample, and we call them the MIRselected sample.

In Table 1 we list the identification number of each source in the MIR-selected sample according to the catalog of Sharma et al. (2011), its celestial coordinates, the estimated source radius, and the flux at $24 \mu \mathrm{m}$. We also show the $8 \mu \mathrm{m}$ flux and the source luminosity in the $\mathrm{H} \alpha$ line and in the near and far continuum UV if sources belong to M 33. The galactocentric distance $\mathrm{R}$, estimated using the tilted ring model, is given in the last column. Only 4 of the 12 sources have detectable FUV emission with luminosities higher than $10^{36} \mathrm{erg} \mathrm{s}^{-1}$, and only two have a weak $\mathrm{H} \alpha$ counterpart. While the lack of $\mathrm{H} \alpha$ is expected for lowmass YSCC because of the IMF incompleteness (Corbelli et al. 2009), the paucity of FUV emission suggests that some source might be associated with embedded YSCCs, distant background galaxies, or QSOs. Most of the 18 sources that are tagged as disk galaxies have detectable FUV emission.

In Fig. 4 we show the location of the MIR sample over the $24 \mu \mathrm{m}$ and FUV maps of M33. The squares indicate the MIR sources that were selected for pointed $\mathrm{CO}$ observations, while the asterisk in the squares highlights sources with detected $\mathrm{CO}$ emission as described in the next section. In the left panel of Fig. 4 we use orange asterisks to indicate the location of the GMCs in the SF disk. The ellipse encloses the SF disk area and has been drawn for $\mathrm{PA}=23^{\circ}$ and $i=54^{\circ}$ (de Vaucouleurs et al. 1991). Selected sources may have galactocentric distances that differ from what the ellipse position suggests because of the change in orientation of the disk beyond $7 \mathrm{kpc}$.

In Fig. 5 we plot the location of selected MIR sources over a smoothed version of the $21 \mathrm{~cm}$ line map (equivalent beam $F W H M=130 \operatorname{arcsec}^{2}$ ) in order to recover emission from the outer disk. The atomic gas map shows that even though all sources are located beyond the SF edge of M33, the majority of them still overlap the bright atomic disk, before the $21 \mathrm{~cm}$ surface brightness drops and the faint warped outer disk takes place. The dark contour level is at $5 \mathrm{Jy} \mathrm{beam}^{-1} \mathrm{~km} \mathrm{~s}^{-1}$, which correspond to a face-on value column density of about $2 \times 10^{20} \mathrm{~cm}^{-2}$. Most of the sources lie in regions of local HI overdensities, which can shield molecules from the weak interstellar dissociating radiation field. Metallicities (expressed as $12+\log (\mathrm{O} / \mathrm{H})$ ) are expected to be roughly a factor 3-4 below solar at galactocentric distances of the sources and imply a CO-to- $\mathrm{H}_{2}$ conversion factor larger than the Galactic in the solar neighborhood (Amorín et al. 2016), that is, between 1.3 and 2 times the conversion factor used for the SF disk (which reads $X_{\mathrm{CO}}=4 \times 10^{20} \mathrm{~K}^{-1} \mathrm{~km}^{-1} \mathrm{~s} \mathrm{~cm}^{-2}$, Gratier et al. 2017).

\section{CO observations}

In this section we describe the observations of $\mathrm{CO} J=1-0$ and CO $J=2-1$ lines at the position of the selected MIR sources in the outer disk. We also present a $5 \operatorname{arcmin}^{2}$ map of the CO $J=2-1$ emission around a bright HII region in the southern side of the outer disk. We refer to this area as the southern field.

\subsection{Pointed observations of the MIR-selected sample}

We searched for CO emission using the IRAM-30 m telescope at the location of all sources in Table 1 . The CO $J=1-0$ and $J=2-1$ lines were observed during March 2016 with an FWHM beam of 21.4 arcsec at $115 \mathrm{GHz}$ and of 10.7 arcsec at $230 \mathrm{GHz}$. The $J=2-1$ line is less likely to be affected by beam dilution than the $J=1-0$ line, but so far out in the disk, the excitation conditions may favor the lower transition. We observed all sources using the wobbler switching mode, making sure not to have $\mathrm{CO}$ emission in the reference beam. The FTS backend with a spectral resolution of $195 \mathrm{kHz}$ was used, corresponding to channel widths of $0.5 \mathrm{~km} \mathrm{~s}^{-1}$ at $115 \mathrm{GHz}$ and $0.25 \mathrm{~km} \mathrm{~s}^{-1}$ at $230 \mathrm{GHz}$. The VESPA backend system with $78.1 \mathrm{kHz}$ resolution $\left(0.2 \mathrm{~km} \mathrm{~s}^{-1}\right)$ was also used at $115 \mathrm{GHz}$, but the noise level was higher than for the FTS backend when data were smoothed at the same spectral resolution, therefore we only discuss the FTS data. All spectra were smoothed to a spectral resolution of $0.5 \mathrm{~km} \mathrm{~s}^{-1}$, and data from both polarizations were averaged. The beam and forward efficiencies used to convert antenna temperatures into main-beam temperatures are 0.71 and 0.95 at $115 \mathrm{GHz}$, respectively, and 0.65 and 0.92 at $230 \mathrm{GHz}$, respectively.

Table 2 summarizes the CO $J=1-0$ and $J=2-1$ data. The identification number of the MIR source in each pointed observation is in given in Col. 1. In Cols. 2 and 3 we show the integrated line brightness per beam I of the $J=1-0$ and $J=2-1$ lines, respectively, in main-beam temperature units. In Cols. 4 and 5 we display the mean velocity $V$ of the two 

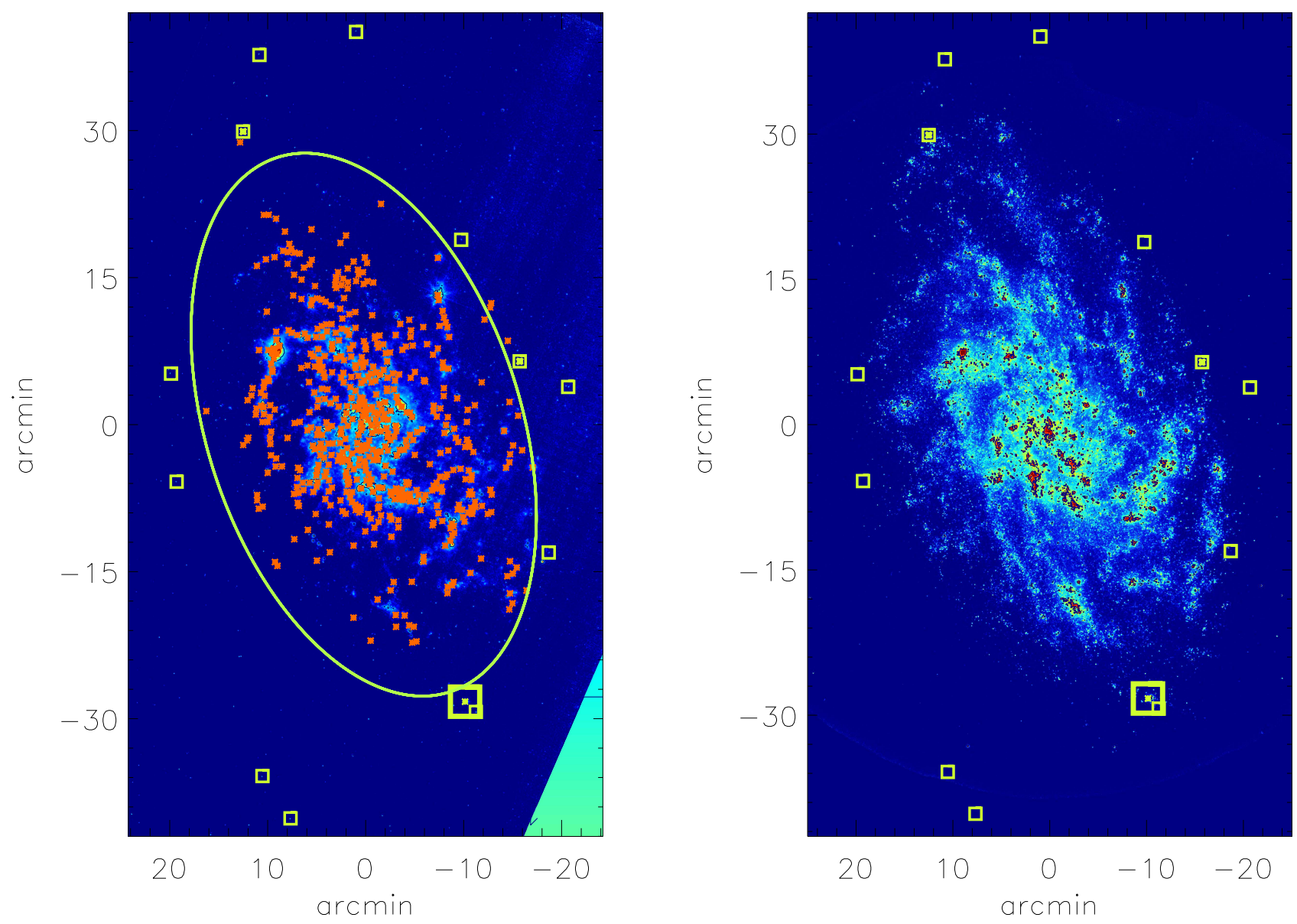

Fig. 4. GMCs detected through the all-disk IRAM survey are plotted with orange asterisks over the $24 \mu \mathrm{m}$ image of M 33 in the left panel. The ellipse encloses the SF disk, with a radius of $7 \mathrm{kpc}$, mapped in the CO $J=2-1$ line by the IRAM all-disk survey. Small open squares indicate the selected MIR sources for the deeper CO pointed observations described in this paper, and the large open square indicates the mapped southern field. Asterisks inside open squares highlight the location of the new detected molecular clouds presented in this paper. They are plotted over the $24 \mu \mathrm{m}$ image of M33 in the left panel and over the FUV-GALEX image of M 33 in the right panel.

lines, and in Cols. 6 and 7, we list the relative line widths $W$. The peak intensity value $P$ is given in Cols. 8 and 9 , and the rms noise level for the CO $J=1-0$ and $J=2-1$ spectra, smoothed at $2 \mathrm{~km} \mathrm{~s}^{-1}$ resolution, is presented in Cols. 10 and 11 , respectively. The $I, V$, and $W$ values are those of the best Gaussian fit to the line after smoothing the data to a resolution of $0.5 \mathrm{~km} \mathrm{~s}^{-1}$. The integrated line brightness obtained by summing the flux in each channel inside the signal spectral window is given in parentheses, and it can be seen that the difference with the integral of the Gaussian fitted line is on the same order as the fit uncertainty. Using the rms noise, we have estimated $4 \mathrm{rms}$ upper limits for the integrated line emission at locations of sources with no detectable signal. The values quoted in Table 2 are for a $8 \mathrm{~km} \mathrm{~s}^{-1}$ spectral window. By requiring a $4 \mathrm{rms}$ detection and for a typical rms value of $5 \mathrm{mK}$, we have that $I_{1-0}<$ $80 \mathrm{mK} \mathrm{km} \mathrm{s}^{-1}$. Similarly, using a typical rms value of $4 \mathrm{mK}$ for the CO $J=2-1$ spectrum, we have that $I_{2-1}<64 \mathrm{mK} \mathrm{km} \mathrm{s}^{-1}$ for undetected emission. The individual upper limits of the integrated line brightness for each observed position are given in Table 2.

In Fig. 6 we show the detected lines. Only 2 out of the 12 MIR sources were detected, and the peak velocities of the $J=1-0$ and $J=2-1$ correspond to within $0.5 \mathrm{~km} \mathrm{~s}^{-1}$, therefore we can assume that the two lines come from the same cloud.
The lines are narrow, and the $J=2-1$ to the $J=1-0$ integrated line brightness ratio varies: it is 0.5 for $\mathrm{s} 799$ and 1.2 for $\mathrm{s} 892$. The small size of the MIR source and the high $J=2-1 / 1-0$ ratio with respect to the standard 0.8 value (Druard et al. 2014) suggests that beam dilution affects source s892. On the other hand, the weakness of the radiation field in these outer regions can easily cause the $\mathrm{CO} J=2-1$ line to be dimmer and lower the $J=2-1$ to $J=1-0$ ratio, as is observed for source s799 (see next section for more details on models for the detected gas).

We also pointed the telescope at five positions close to source s892 (s892-o1, o2, o3, o4, and o5) whose offsets in arcsec for RA and Dec are $(11.5,-1.0),(-10.5,-11.4),(18,11.4)$, $(-36,22.5)$, and $(63,36.2)$, and one position close to source 5555 ( $5555-01)$, whose offset is $(7,-7)$. No lines were detected in any of the offset locations. The upper limits are on the same order as the fluxes detected on source at $(0,0)$ offset, and we conclude that the cloud associated with source s 892 does not have brighter peaks in nearby locations. This is supported by the null detection after stacking the offset spectra, as discussed in the next section. The position of source s555 is in the lower right corner of the southern field, as discussed in the next subsection, and its upper limit confirms that the emission in the southern field is not very extended. 


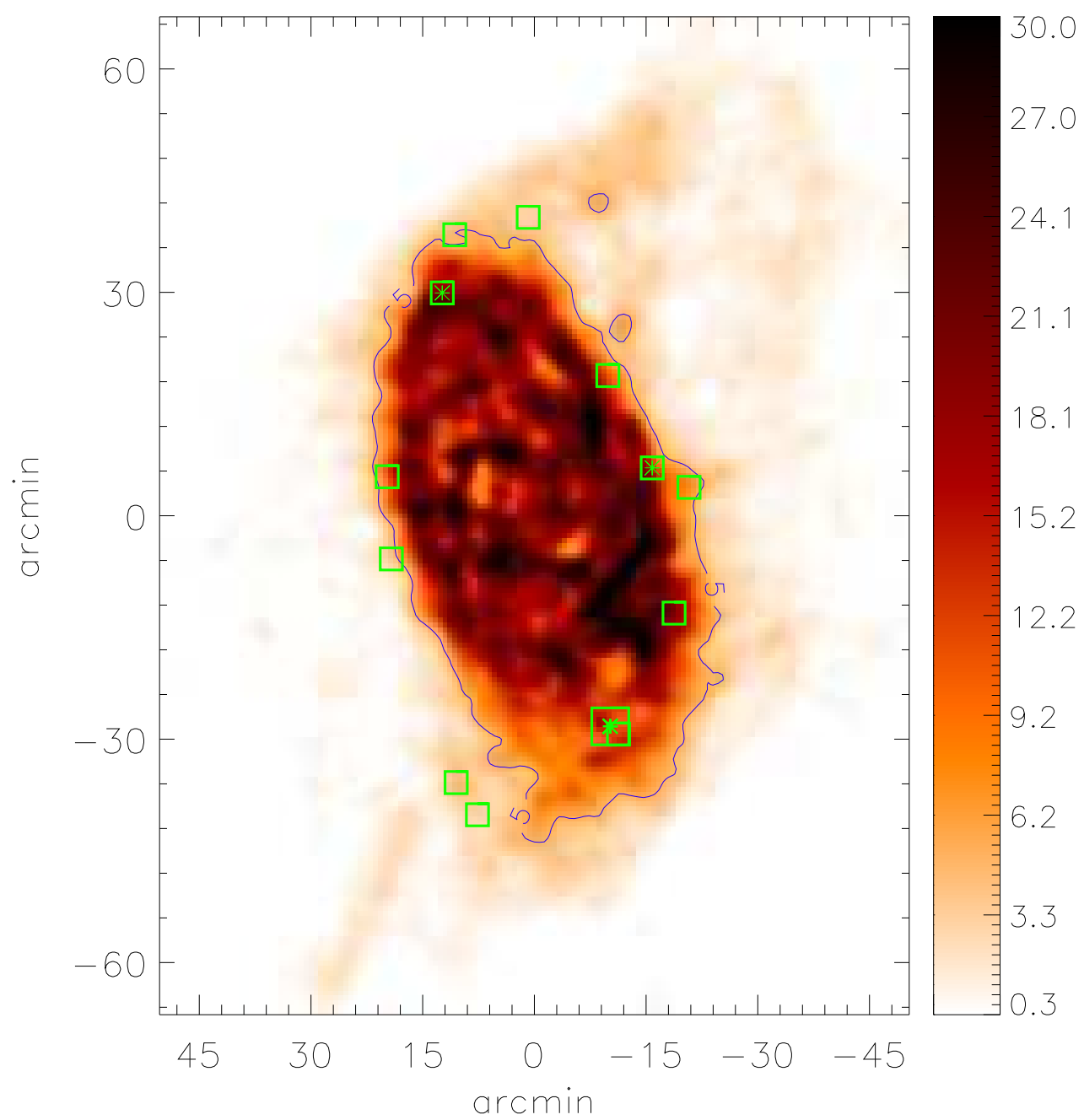

Fig. 5. Location of sources in the MIRselected sample (open squares) plotted over the $21 \mathrm{~cm}$ map of M33. Asterisks indicate the location of MIR sources where $\mathrm{CO}$ has been detected. The color bar and map unit are Jy beam ${ }^{-1} \mathrm{~km} \mathrm{~s}^{-1}$; a 130 arcsec beam has been used for this smoothed $21 \mathrm{~cm}$ map. The dark contour level at $5 \mathrm{Jy}$ beam ${ }^{-1} \mathrm{~km} \mathrm{~s}^{-1}$ corresponds to a face-on HI column density of about $2 \times 10^{20} \mathrm{~cm}^{-2}$

\subsection{Map of the southern field}

A region centered at $\mathrm{RA}=01: 33: 06$ Dec $=30^{\circ} 11^{\prime} 30^{\prime \prime}$ $\left(\mathrm{RA}=23.2750^{\circ} \mathrm{Dec}=30.1917^{\circ}\right)$, close to the position of a large HII region in the extreme south of M33, was included in the $\mathrm{CO} J=2-1$ observations of the all-disk survey (Druard et al. 2014). This region was observed separately because the position of the center of M 33 in the sky exceeds the IRAM $83^{\circ}$ elevation limit for about 40 min every day. Defining the southern region as a separate source enabled us to observe it during about 25 of the 40 min that would have been lost each day (the remaining time was used to check pointing). It was observed at $82.8^{\circ}$ elevation on average and is slightly beyond the southern limit of the map presented in Druard et al. (2014). It was therefore not presented in Druard et al. (2014).

The bright HII region in the southern field, also known as MA1 (Mayall \& Aller 1942), lies at a galactocentric distance of $7.4 \mathrm{kpc}$, and it is one of the few HII regions that are located beyond the drop of the average $\mathrm{H} \alpha$ surface brightness. The metal content of this outer HII region has been investigated, and the most recent estimate for its oxygen abundance is $12+\log (\mathrm{O} / \mathrm{H})=8.28 \mathrm{dex}$ (Magrini et al. 2010). This HII region is coincident with the MIR source identified by Sharma et al. (2011) with ID number 562, which has a flux of $9.2 \mathrm{mJy}$ and a size of $4.4 \operatorname{arcsec}$ at $24 \mu \mathrm{m}$. It hosts a stellar cluster whose stellar mass and age have been estimated to be about $6000 M_{\odot}$ and $8 \mathrm{Myr}$ by Sharma et al. (2011). This HII region is much brighter than any other in the MIR sample selected in the outer disk.

The southern field of M33 was observed as described in Druard et al. (2014), using on-the-fly mapping in $\mathrm{CO}(2-1)$ with the HERA multibeam receiver. Figure 7 shows the $\mathrm{H} \alpha$ emission in color with the $100 \mu \mathrm{m}$ PACS emission in black contours and the positions where $\mathrm{CO}$ emission is well above the noise. The thick red contour indicates the extent of the $\mathrm{CO}$ map. The four $\mathrm{CO}$ spectra with a high enough signal-to-noise ratio are overplotted, and the triangles indicate the corresponding position in the disk. Spectra within 5 arcsec of the nominal position were summed to improve the signal-to-noise ratio. This broadens the beam only slightly since these spectra are all within the halfpower size of a single beam. The nominal position of the spectra is given by the pixel with the highest signal-to-noise ratio. A white contour shows where $N_{\mathrm{HI}}=1.8 \times 10^{21} \mathrm{~cm}^{-2}$, that is, where the HI column density is high. In the southern field the HI column density peaks in between the two SF regions and not where star formation is taking place, which is likely due to the transition from $\mathrm{HI}$ to $\mathrm{H}_{2}$ and to the expansion of the HII region shell, which compresses the surrounding neutral ISM and thus enhances the local density.

Each box in Fig. 7 shows the $\mathrm{CO}(2-1)$ spectrum in white and the HI line temperature (divided by 1000) in light blue. The $\mathrm{CO}$ spectra are presented on the $T_{\mathrm{mb}}$ scale and all spectra are in Kelvin. The $\mathrm{CO}$ emission is strongest close to the HII region center. All CO line widths are typical of single GMC, although they 
Table 1. Properties of the MIR-selected sample.

\begin{tabular}{|c|c|c|c|c|c|c|c|c|c|}
\hline $\begin{array}{l}\text { ID } \\
\ldots \\
\ldots\end{array}$ & $\begin{array}{l}\text { RA } \\
\text { deg }\end{array}$ & $\begin{array}{l}\text { Dec } \\
\text { deg }\end{array}$ & $\begin{array}{c}r \\
\operatorname{arcsec}\end{array}$ & $\begin{array}{l}F_{24} \\
\mathrm{mJy}\end{array}$ & $\begin{array}{c}F_{8} \\
\mathrm{mJy}\end{array}$ & $\begin{array}{c}\log L_{\mathrm{H} \alpha} \\
\operatorname{erg~s}^{-1}\end{array}$ & $\begin{array}{c}\log L_{\mathrm{FUV}} \\
\mathrm{erg} \mathrm{s}^{-1}\end{array}$ & $\begin{array}{c}\log L_{\mathrm{NUV}} \\
\mathrm{erg} \mathrm{s}^{-1}\end{array}$ & $\begin{array}{c}\mathrm{R} \\
\mathrm{kpc}\end{array}$ \\
\hline s537 & 23.6099 & 29.9906 & 1.8 & $1.29 \pm 0.02$ & $0.14 \pm 0.02$ & . & & & 10.4 \\
\hline s542 & 23.6651 & 30.0625 & 1.7 & $1.31 \pm 0.02$ & $0.48 \pm 0.03$ & & & & 10.0 \\
\hline s555 & 23.2454 & 30.1715 & 1.8 & $1.07 \pm 0.02$ & $0.33 \pm 0.03$ & & & & 7.8 \\
\hline s631 & 23.1010 & 30.4421 & 1.3 & $0.39 \pm 0.02$ & $0.35 \pm 0.03$ & $\ldots$ & 36.6 & 36.3 & 7.9 \\
\hline s672 & 23.8356 & 30.5626 & 1.6 & $0.83 \pm 0.02$ & $0.73 \pm 0.04$ & .. & $\ldots$ & $\ldots$ & 8.9 \\
\hline s771 & 23.0613 & 30.7235 & 1.7 & $0.87 \pm 0.02$ & $0.49 \pm 0.03$ & & & & 9.2 \\
\hline s787 & 23.8474 & 30.7463 & 2.0 & $2.62 \pm 0.02$ & - & $\ldots$ & $\ldots$ & & 8.5 \\
\hline s799 & 23.1572 & 30.7673 & 1.4 & $0.54 \pm 0.01$ & $0.33 \pm 0.03$ & 34.7 & 37.3 & 37.0 & 7.8 \\
\hline s854 & 23.2726 & 30.9742 & 1.4 & $0.45 \pm 0.01$ & $0.26 \pm 0.02$ & $\ldots$ & 36.8 & 36.9 & 7.7 \\
\hline s892 & 23.7057 & 31.1582 & 1.4 & $0.45 \pm 0.01$ & $0.37 \pm 0.03$ & 35.7 & 37.8 & 37.5 & 8.1 \\
\hline s905 & 23.6735 & 31.2885 & 1.9 & $2.77 \pm 0.02$ & $1.80 \pm 0.06$ & $\ldots$ & $\ldots$ & $\ldots$ & 10.1 \\
\hline s907 & 23.4811 & 31.3279 & 2.0 & $1.94 \pm 0.02$ & $0.43 \pm 0.03$ & $\ldots$ & $\ldots$ & $\ldots$ & 9.8 \\
\hline
\end{tabular}

Notes. The identification number of each source in the MIR-selected sample is listed in Col. 1, and the relative celestial coordinates are presented in Cols. 2 and 3. The estimated source radius is given in Col. 4 and the flux at $24 \mu \mathrm{m}$ in Col. 5. The $8 \mu \mathrm{m}$ flux is listed in Col. 6, and the source luminosity in the $\mathrm{H} \alpha$ line and in the near and far continuum UV are given in Cols. 7-9, respectively. In the last column we show the source galactocentric distance.

Table 2. CO line pointed observations of the MIR-selected sample and Gaussian fits to detected lines.

\begin{tabular}{|c|c|c|c|c|c|c|c|c|c|c|}
\hline $\begin{array}{l}\text { ID } \\
\ldots\end{array}$ & $\begin{array}{c}\quad I_{1-0} \\
\mathrm{mK} \mathrm{km} \mathrm{s}^{-1}\end{array}$ & $\begin{array}{c}I_{2-1} \\
\mathrm{mK} \mathrm{km} \mathrm{s}^{-1}\end{array}$ & $\begin{array}{c}V_{1-0} \\
\mathrm{~km} \mathrm{~s}^{-1}\end{array}$ & $\begin{array}{c}V_{2-1} \\
\mathrm{~km} \mathrm{~s}^{-1}\end{array}$ & $\begin{array}{c}W_{1-0} \\
\mathrm{~km} \mathrm{~s}^{-1}\end{array}$ & $\begin{array}{c}W_{2-1} \\
\mathrm{~km} \mathrm{~s}^{-1}\end{array}$ & $\begin{array}{c}P_{1-0} \\
\mathrm{mK}\end{array}$ & $\begin{array}{c}P_{2-1} \\
\mathrm{mK}\end{array}$ & $\begin{array}{c}\mathrm{rms}_{1-0} \\
\mathrm{mK}\end{array}$ & $\begin{array}{c}\mathrm{rms}_{2-1} \\
\mathrm{mK}\end{array}$ \\
\hline s537 & $<77$ & $<49$ & $\ldots$ & $\ldots$ & $\ldots$ & $\ldots$ & $\cdots$ & & 4.8 & 3.1 \\
\hline $\mathrm{s} 542$ & $<93$ & $<70$ & $\ldots$ & $\ldots$ & $\ldots$ & $\ldots$ & $\ldots$ & $\ldots$ & 5.8 & 4.3 \\
\hline s555 & $<75$ & $<75$ & $\ldots$ & $\ldots$ & $\ldots$ & $\ldots$ & $\ldots$ & $\ldots$ & 4.7 & 4.7 \\
\hline s555-o1 & $<140$ & $<117$ & $\ldots$ & $\ldots$ & $\ldots$ & $\ldots$ & $\ldots$ & $\ldots$ & 8.7 & 7.3 \\
\hline s631 & $<75$ & $<43$ & $\ldots$ & $\ldots$ & $\ldots$ & $\ldots$ & $\ldots$ & $\ldots$ & 4.7 & 2.7 \\
\hline s672 & $<97$ & $<80$ & $\ldots$ & $\ldots$ & $\ldots$ & $\ldots$ & $\cdots$ & $\cdots$ & 6.1 & 5.0 \\
\hline s771 & $<83$ & $<83$ & $\ldots$ & $\ldots$ & $\ldots$ & $\ldots$ & $\ldots$ & $\ldots$ & 5.2 & 5.2 \\
\hline s787 & $<77$ & $<57$ & $\ldots$ & $\ldots$ & $\ldots$ & $\ldots$ & $\ldots$ & $\ldots$ & 4.8 & 3.6 \\
\hline s799 & $408 \pm 45(467)$ & $202 \pm 19(227)$ & -179.1 & -179.5 & 3.7 & 2.4 & 104 & 79 & 8.8 & 4.8 \\
\hline s854 & $<87$ & $<89$ & $\ldots$ & $\ldots$ & $\ldots$ & $\ldots$ & $\ldots$ & $\ldots$ & 5.4 & 5.6 \\
\hline s892 & $150 \pm 23(149)$ & $179 \pm 11(171)$ & -272.4 & -272.8 & 2.2 & 3.0 & 65 & 57 & 6.1 & 4.5 \\
\hline s892-o1 & $<171$ & $<128$ & $\ldots$ & $\ldots$ & $\ldots$ & $\ldots$ & $\ldots$ & $\ldots$ & 10.7 & 8.0 \\
\hline s892-o2 & $<134$ & $<140$ & $\ldots$ & $\ldots$ & $\ldots$ & $\ldots$ & $\ldots$ & $\ldots$ & 8.4 & 8.7 \\
\hline s892-o3 & $<158$ & $<132$ & $\ldots$ & $\ldots$ & $\ldots$ & $\ldots$ & $\ldots$ & $\ldots$ & 9.9 & 8.3 \\
\hline s892-o4 & $<144$ & $<146$ & $\ldots$ & $\ldots$ & $\ldots$ & $\ldots$ & $\ldots$ & $\ldots$ & 9.0 & 9.1 \\
\hline s892-o5 & $<205$ & $<131$ & $\ldots$ & $\ldots$ & $\ldots$ & $\ldots$ & $\ldots$ & $\ldots$ & 12.8 & 8.2 \\
\hline s905 & $<101$ & $<63$ & $\ldots$ & $\ldots$ & $\ldots$ & $\ldots$ & $\ldots$ & $\ldots$ & 6.3 & 3.9 \\
\hline s907 & $<85$ & $<61$ & $\ldots$ & $\ldots$ & $\ldots$ & $\ldots$ & $\ldots$ & $\ldots$ & 5.3 & 3.8 \\
\hline
\end{tabular}

Notes. The ID of the MIR source in each pointed observation is given in Col. 1. In Cols. 2 and 3 we show I, the integrated line brightness per beam of the $J=1-0$ and $J=2-1$ detected lines, respectively (in parentheses we add the values obtained by summing the flux in each channel) or their upper limits. In Cols. 4 and 5 we display the mean velocity $V$ of the detected lines, in Cols. 6 and 7 we list the relative line widths $W$, and in Cols. 8 and 9 the peak intensity values $P$. In the last two columns we show the rms noise level for the CO $J=1-0$ and $J=2-1$ spectra smoothed at $2 \mathrm{~km} \mathrm{~s}^{-1}$ resolution.

are broader than most spectra in the outer disk of M33, as for sources s799 and s892 (but see also Braine et al. 2012), presumably due to the high level of star formation and to the evolution of the HII region. Table 3 gives the integrated intensities and Gaussian fit results in main-beam temperature units. The beam and forward efficiencies used to convert antenna temperatures into main-beam temperatures at the time of these observations are 0.55 and 0.92 at $230 \mathrm{GHz}$. The uncertainties are from the fitting program in CLASS ${ }^{1}$. Uncertainties on the integrated intensities

\footnotetext{
1 See http://www.iram.fr/IRAMFR/GILDAS/
}

in Col. 2 can be calculated as $\Delta I=1.5 \mathrm{rms} \times \sqrt{\mathrm{W}_{2-1} \times 2.6}$ where rms is given in Col. 8 and $W_{2-1}$ in Col. 5. These uncertainties are similar to those in the GaussArea task in CLASS. We did not subtract the $2.6 \mathrm{~km} \mathrm{~s}^{-1}$ channel width, therefore the $W_{2-1}$ values in Col. 5 are upper limits.

\section{Radial decline of the molecular cloud mass in $\mathrm{M} 33$}

In this section we discuss the results of detected and undetected $\mathrm{CO}$ line emission. We averaged spectra of undetected sources to 


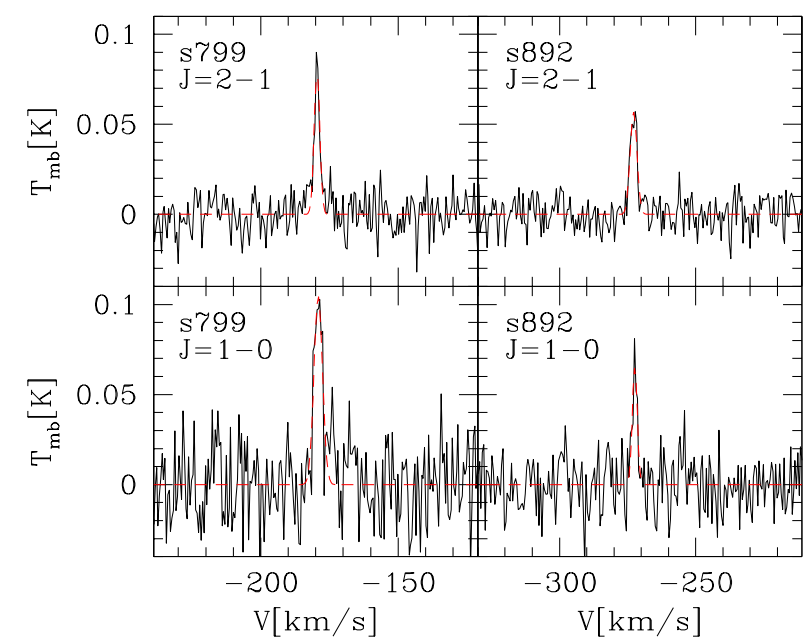

Fig. 6. CO line emission detected at the location of sources s799 and s892 and the best-fit Gaussian function. Velocities are heliocentric, and the line emission is in main-beam temperature units.

further lower the rms and search for very faint $\mathrm{CO}$ lines in the outer disk. We evaluated possible background contamination in the MIR sample, which might be severe for faint sources at large galactocentric distances, in order to understand the paucity of $\mathrm{CO}$ lines in the MIR sample. Finally, we compare some characteristics of the HII region in the southern field, including the CO line intensities, to those of SF sites in the SF disk, which share a similar morphology and MIR emission. This should help us understand whether the outer disk environment or the YSCC evolution plays a major role in shaping the observed properties of MA1.

\subsection{Detected lines and the associated molecular clouds}

The molecular mass at the location of sources s799 and s892 is uncertain because it depends on the extent and position of the clouds with respect to the beam. When clouds are smaller than the FWHM of the $115 \mathrm{GHz}$ beam, as suggested by the lack of $\mathrm{CO}$ detection in the proximity of s892, the measured $\mathrm{CO}$ $J=2-1 / 1-0$ integrated brightness temperature ratio is higher than the intrinsic line ratio $R_{21}$, defined as the $J=2-1 / J=1-0$ line ratio for extended sources because beam dilution is stronger for the $J=1-0$ line. Moreover, the $\mathrm{CO}-$ to- $\mathrm{H}_{2}$ conversion factor can be different than the constant value determined and used for the SF disk. In what follows we estimate cloud masses using different methods, assuming that clouds are centered with respect to the beam and uniform in brightness. Using Eqs. (5), (6), and (8) in Corbelli et al. (2011), we can estimate cloud sizes and masses from the observed $J=2-1 / 1-0$ line ratio, and we refer to this method as the ratio method. In practice, we convolved the cloud surface brightness with the beam for the $J=1-0$ and $J=2-1$ line assuming a cloud size $\mathrm{D}$ and an intrinsic line ratio $R_{21}$. We then inferred $\mathrm{D}$ by equating the expected line ratio to the observed one. The cloud mass was then estimated using a CO-to- $\mathrm{H}_{2}$ conversion factor, $X_{\mathrm{CO}}$, which depends on metallicity. We can also evaluate the cloud size and mass assuming virial equilibrium for a given CO-to- $\mathrm{H}_{2}$ conversion factor. With this method, called the virial method, the cloud size is determined by equating the virial mass to the luminous mass as in Eq. (18) of Corbelli et al. (2011). When the $J=1-0$ and $J=2-1$ CO lines are both detected, $R_{21}$ is estimated. Finally, in the third method, the fill method, we assumed that the source is as extended as the FWHM beam at $230 \mathrm{GHz}$. We computed the mass assuming a $\mathrm{CO}-$ to- $\mathrm{H}_{2}$ conversion factor that depends on metallicity, and derived the cloud $\mathrm{CO}$ surface brightness by equating the observed CO $J=2-1$ line brightness to the expected one using beam convolution. With this method, the intrinsic line ratio $R_{21}$ was estimated only when the $J=1-0$ line was also detected, otherwise $R_{21}$ was an input value.

We estimated the metallicity $Z$ at the location of sources s799 and s892 using the galactocentric distance $R$ and the oxygen abundance radial gradient of Magrini et al. (2010). Following Amorín et al. (2016), we assumed a $Z^{-1.5}$ metallicity dependence of $X_{\mathrm{CO}}$ and write

$\log \frac{X_{\mathrm{CO}}}{2 \times 10^{20}}=12.85-1.5\left(12+\log \frac{O}{H}\right)=0.1+0.066 R$.

For $R=3 \mathrm{kpc}$, this formula gives $X_{\mathrm{CO}}=4 \times 10^{20} \mathrm{~K}^{-1}$ $\mathrm{km}^{-1} \mathrm{~s} \mathrm{~cm}^{-2}$, the commonly used conversion factor for the SF disk of M33, and hence it well approximates the average value of $X_{\mathrm{CO}}$ derived for the inner and intermediate disk by Gratier et al. (2017). These authors found that for M 33 the COto- $\mathrm{H}_{2}$ conversion factor is independent of radius out to about $6 \mathrm{kpc}$, but beyond this radius, the lack of GMCs prevents any definitive conclusion.

We list in Table 4 the estimated molecular cloud parameters for the two detected sources in the MIR sample using the three methods. Estimated cloud masses include He and heavier elements. For the ratio method we used three different values of $R_{21}$ and quote the results when a value of the cloud size $D$ satisfies the assumptions (i.e., that gives the observed $J=1-0$ and $J=2-1$ integrated line brightness for the assumed intrinsic ratio $R_{21}$ ). For source $\mathrm{s} 892$, all methods give similar cloud mass estimates, on the order of $2 \times 10^{4} M_{\odot}$, while the cloud extent is not well determined. The low $I_{2-1} / I_{1-0}$ ratio observed at the location of source s799 might be due to the low-excitation conditions of the gas, because the SF source is weak in FUV and $\mathrm{H} \alpha$ emission. At this location, the cloud mass can be as high as $10^{5} M_{\odot}$ and more extended than the beam FWHM at $115 \mathrm{GHz}$. Smaller sizes and lower masses are inferred using the virial or the fill method, which predict low values of $R_{21}$. The expected stellar masses of YSCCs associated with sources s799 and s892 are $200-300 M_{\odot}$, as determined by the spectral energy distribution fits (Sharma et al. 2011), and they imply a total stellar cluster-to-cloud mass ratio lower than 0.02 .

The molecular cloud masses associated with sources s799 and s892, although very uncertain, confirm the paucity of giant molecular complexes beyond the SF edge and the decrease in mean value of molecular cloud mass from the intermediate to the outer disk. We underline that the results shown in Table 4 are relative to a CO-to- $\mathrm{H}_{2}$ conversion factor that is $8.2 \times 10^{20}$ and $8.6 \times 10^{20} \mathrm{~K}^{-1} \mathrm{~km}^{-1} \mathrm{~s} \mathrm{~cm}^{-2}$ for sources $\mathrm{s} 799$ and $\mathrm{s} 892$, respectively. These values are about a factor 2 higher than that inferred for the SF disk by Gratier et al. (2017) and used by Corbelli et al. (2017) and by Braine et al. (2018). Although beyond the SF edge the conversion factor may increase with respect to the SF disk as a result of more extreme physical and chemical conditions, it is unlikely that molecular clouds associated with sources s799 and s892 are much more massive than what we quote in Table 3. This implies that the average mass and size of perturbations that occasionally grow in the outer disk drop beyond the SF edge. The low mass of the YSCCs that are hosted by these clouds provides additional support to this conclusion.

Using the virial and fill method, we also quote in Table 4 the cloud mass at the four positions listed in Table 3 in the southern field. The metallicity was determined for MA1 


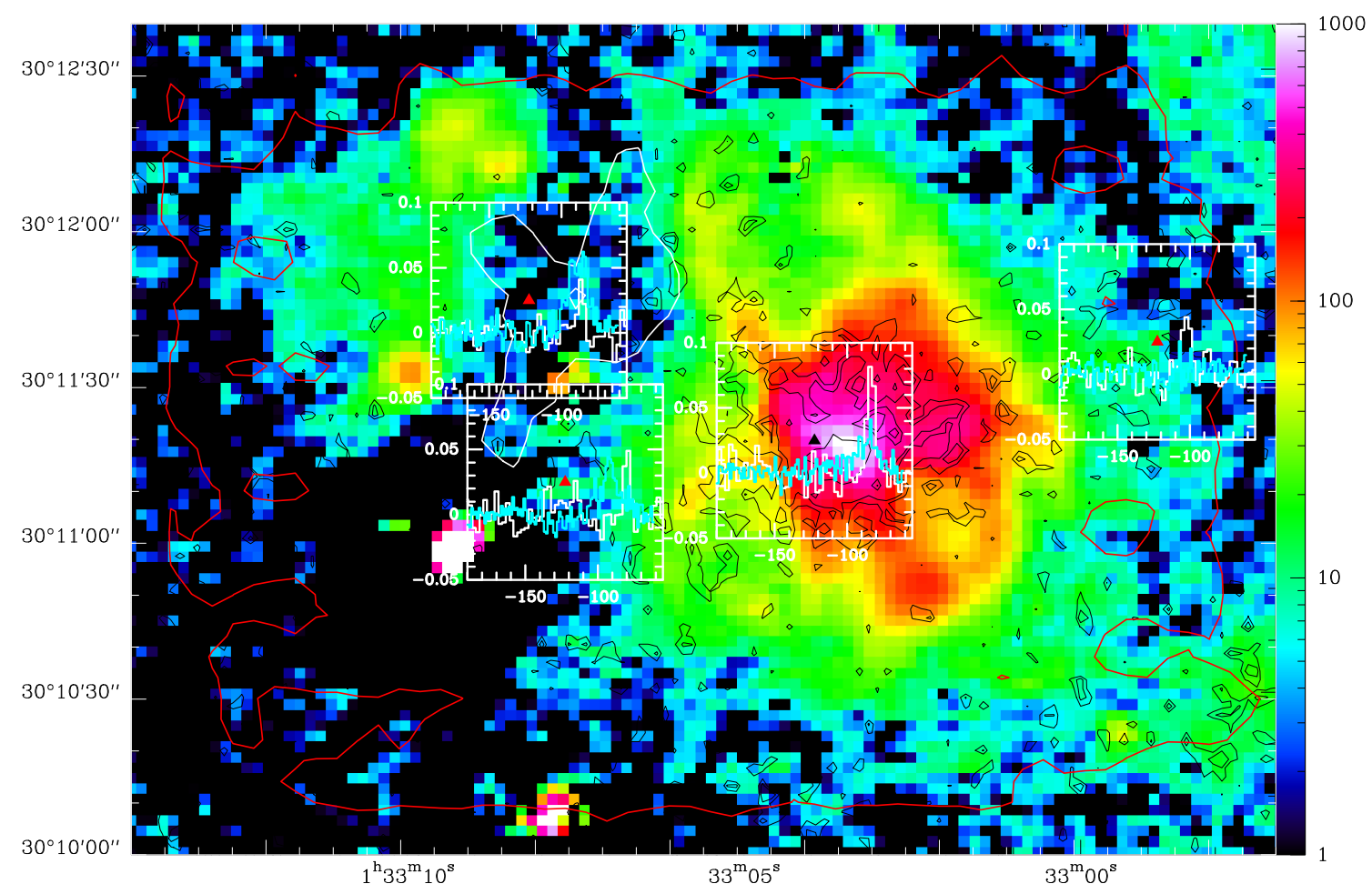

Fig. 7. Southern field $\mathrm{H} \alpha$ emission in color, with $100 \mu \mathrm{m}$ PACS emission in black contours. Four CO spectra in the southern field where the signal is well above the noise are shown, with the precise positions indicated with red or black triangles. The $21 \mathrm{~cm}$ spectrum at the location of the detected CO line is shown in cyan. The thick red contour indicates the extent of the CO map. The white contour between the H $\alpha$ emitting zones shows the $N_{\mathrm{HI}}=1.8 \times 10^{21} \mathrm{~cm}^{-2}$ column density level (in the rest of the region, the HI column densities are lower than the contour value).

Table 3. Gaussian fits to the CO $J=2-1$ integrated line brightness per beam for the detected emission in the southern field.

\begin{tabular}{lccccccc}
\hline \hline $\begin{array}{l}\text { Name } \\
\ldots\end{array}$ & $\begin{array}{c}\text { Offset } \\
\operatorname{arcsec}\end{array}$ & $\begin{array}{c}I_{2-1} \\
\mathrm{mK} \mathrm{km} \mathrm{s}^{-1}\end{array}$ & $\begin{array}{c}V_{2-1} \\
\mathrm{~km} \mathrm{~s}^{-1}\end{array}$ & $\begin{array}{c}W_{2-1} \\
\mathrm{~km} \mathrm{~s}^{-1}\end{array}$ & $\begin{array}{c}P_{2-1} \\
\mathrm{mK}\end{array}$ & $\begin{array}{c}\Delta V_{2-1} \\
\mathrm{~km} \mathrm{~s}^{-1}\end{array}$ & $\begin{array}{c}\mathrm{rms}_{2-1} \\
\mathrm{mK}\end{array}$ \\
\hline $562 \mathrm{a}$ & $(-562,-1704)$ & $427 \pm 84(375)$ & $-79.9 \pm 0.4$ & $4.3 \pm 1.1$ & 94 & $-83,-77$ & 16.9 \\
$562 \mathrm{~b}$ & $(-676,-1677)$ & $595 \pm 104(492)$ & $-102.4 \pm 0.6$ & $7.2 \pm 1.4$ & 77 & $-107,-98$ & 16.1 \\
$562 \mathrm{c}$ & $(-610,-1696)$ & $927 \pm 100(825)$ & $-83.6 \pm 0.3$ & $6.0 \pm 0.7$ & 144 & $-89,-80$ & 17.2 \\
$562 \mathrm{~d}$ & $(-555,-1669)$ & $341 \pm 67(336)$ & $-86.5 \pm 0.5$ & $4.5 \pm 0.9$ & 70 & $-91,-82$ & 13.4 \\
\hline
\end{tabular}

Notes. The name of detected cloud clump is given in Col. 1. Column 2 gives the cloud clump offset with respect to the center of M 33 . Column 3 gives the $\mathrm{CO}$ line intensity in main-beam temperature units computed as the area of the best-fit Gaussian function, in parentheses we list the values obtained by summing the flux in each channel of the selected spectral window. Columns 4 and 5 give the central velocity and the line width of the Gaussian fits, respectively. The signal peak and spectral window are given in Cols. 6 and 7, respectively. Column 8 gives the per channel noise level of the spectra (for a channel width of $2.6 \mathrm{~km} \mathrm{~s}^{-1}$ ).

in the southern field to be $12+\log (\mathrm{O} / \mathrm{H})=8.28$ (Magrini et al. 2010 ), and this implies a CO-to- $\mathrm{H}_{2}$ conversion factor $X_{\mathrm{CO}}=5.4$ $10^{20} \mathrm{~K}^{-1} \mathrm{~km}^{-1} \mathrm{~s} \mathrm{~cm}^{-2}$, according to the above equation. The strong radiation field of the HII region in the southern field suggests that very low values of $R_{21}$ are excluded. The total molecular hydrogen mass estimated for $R_{21}=1.2$ in the southern field is very similar for both the virial and the fill method, being $5.4 \times 10^{4}$ and $6.1 \times 10^{4} M_{\odot}$, respectively. The total molecular hydrogen mass is in agreement with the mass of other GMCs measured in the outer disk and plotted in Fig. 1. The virial method implies more compact clouds, but as discussed later in this section, virial equilibrium is unlikely for gas in the proximity of an HII region that is not compact. Radiation from the stellar cluster breaks through the original cloud, which is swept away by the expanding ionized shell. Given the linear scaling of cloud mass with $1 / R_{21}$ for the fill methods, we estimate a total molecular mass of about $10^{5} M_{\odot}$ for $R_{21}=0.8$, which is still in agreement with a drop of the mean molecular cloud mass beyond the SF disk.

\subsection{Stacking spectra}

The ratio of the integrated CO $J=1-0$ line brightness to the $24 \mu \mathrm{m}$ source flux, $I_{1-0} / F_{24}$, is 0.76 and $0.33 \mathrm{~K} \mathrm{~km} \mathrm{~s}^{-1} \mathrm{mJy}^{-1}$ for sources s799 and s892, respectively. These values are higher than those in the sample of Corbelli et al. (2011) for brighter MIR sources in the SF disk $\left(0.05<I_{1-0} / F_{24}<0.28 \mathrm{~K} \mathrm{~km} \mathrm{~s}^{-1} \mathrm{mJy}^{-1}\right)$. For undetected sources of the MIR sample presented in this paper, this ratio is always lower than $0.2 \mathrm{~K} \mathrm{~km} \mathrm{~s}^{-1} \mathrm{mJy}^{-1}$ and hence well below the values for the two detected sources in the outer disk. To further lower this limit, given the lower CO-to$24 \mu \mathrm{m}$ flux ratios measured by Corbelli et al. (2011) in the SF disk, we stacked the CO spectra of MIR sources with no detected lines. 
Table 4. Estimated properties of the detected molecular clouds in the outer disk for a metallicity-dependent $\mathrm{CO}$-to- $\mathrm{H}_{2}$ conversion factor.

\begin{tabular}{cccccc}
\hline \hline ID & Line & $R_{21}$ & $\begin{array}{c}D \\
\operatorname{arcsec}\end{array}$ & $\begin{array}{c}M \\
10^{4} M_{\odot}\end{array}$ & Method \\
\hline 799 & $2-1,1-0$ & 0.4 & 34 & 9.9 & Ratio \\
799 & $1-0$ & 0.15 & 9.2 & 5.1 & Virial \\
799 & $2-1$ & 0.15 & 9.2 & 2.2 & Virial \\
799 & $2-1,1-0$ & 0.26 & 11 & 5.2 & Fill \\
892 & $2-1,1-0$ & 0.4 & 12 & 2.1 & Ratio \\
892 & $2-1,1-0$ & 0.8 & 27 & 3.0 & Ratio \\
892 & $1-0$ & 0.37 & 10 & 2.0 & Virial \\
892 & $2-1$ & 0.32 & 5.2 & 1.9 & Virial \\
892 & $2-1,1-0$ & 0.63 & 11 & 2.0 & Fill \\
$562 \mathrm{a}$ & $2-1$ & 0.8 & 2.0 & 1.5 & Virial \\
$562 \mathrm{~b}$ & $2-1$ & 0.8 & 1.0 & 2.1 & Virial \\
$562 \mathrm{c}$ & $2-1$ & 0.8 & 2.2 & 3.3 & Virial \\
$562 \mathrm{~d}$ & $2-1$ & 0.8 & 1.4 & 1.2 & Virial \\
$562 \mathrm{a}$ & $2-1$ & 1.2 & 1.3 & 1.0 & Virial \\
$562 \mathrm{~b}$ & $2-1$ & 1.2 & 0.7 & 1.4 & Virial \\
$562 \mathrm{c}$ & $2-1$ & 1.2 & 1.5 & 2.2 & Virial \\
$562 \mathrm{~d}$ & $2-1$ & 1.2 & 1.0 & 0.8 & Virial \\
$562 \mathrm{a}$ & $2-1$ & 1.2 & 11 & 1.1 & Fill \\
$562 \mathrm{~b}$ & $2-1$ & 1.2 & 11 & 1.6 & Fill \\
$562 \mathrm{c}$ & $2-1$ & 1.2 & 11 & 2.5 & Fill \\
$562 \mathrm{~d}$ & $2-1$ & 1.2 & 11 & 0.9 & Fill \\
\hline & & & & & \\
\hline
\end{tabular}

Notes. The CO rotational line used to infer the cloud parameters is given in Col. 2, the intrinsic CO line ratio in Col. 3, and the cloud diameter in Col. 4. The cloud mass is shown in Col. 5, and in the last column, we indicate the method used to derive the cloud parameters.

We applied the stacking procedure to all $\mathrm{CO}$ spectra with an offset near source s892 and independently to the ten $\mathrm{CO}$ spectra with undetected line emission centered at the position of MIR sources. We performed this for the CO $J=1-0$ and $J=2-1$ line separately by computing the expected frequency of the $\mathrm{CO}$ lines for each source. We aligned the spectra according to the expected frequencies and averaged them. We estimated the expected line frequencies from the $21 \mathrm{~cm}$ line velocity because $21 \mathrm{~cm}$ emission is present at all selected positions in the disk. Despite the very low noise in the stacked spectra, no CO lines were detected. The stacked spectra relative to the ten undetected sources for the $\mathrm{CO}$ $J=1-0$ and $J=2-1$ lines are shown in Fig. 8. The rms is as low as $1 \mathrm{mK}$ for the $J=2-1$ line and is $1.5 \mathrm{mK}$ for the $J=1-0$ line. The dashed line in Fig. 8 indicates the location of the expected line. We have to underline that the detected CO lines in the MIR sample are very narrow and this increases the difficulty of the stacking technique given the velocity resolution of $1.25 \mathrm{~km} \mathrm{~s}^{-1}$ in the HI database and the possible shift of molecular gas velocities by a few $\mathrm{km} \mathrm{s}^{-1}$ with respect to the velocities of the diffuse atomic gas.

The low rms of the stacked spectra implies that for a $8 \mathrm{~km} \mathrm{~s}^{-1}$ spectral window, the average ratio of the integrated $\mathrm{CO} J=$ 1-0 line brightness to the $24 \mu \mathrm{m}$ source flux is lower than $0.02 \mathrm{~K} \mathrm{~km} \mathrm{~s}^{-1} \mathrm{mJy}^{-1}$. This upper limit is more than a factor 10 lower than the measured CO-to- $24 \mu \mathrm{m}$ flux ratio for the two MIR sources detected in the outer disk and lower than any measured CO-to-24 $\mu \mathrm{m}$ flux ratio in the whole disk of M 33. The lack of $\mathrm{CO}$ detection for most of the sources in the MIR sample casts doubts on the association of the same faint MIR sources with the M33 disk, and we address this question in the next subsection.

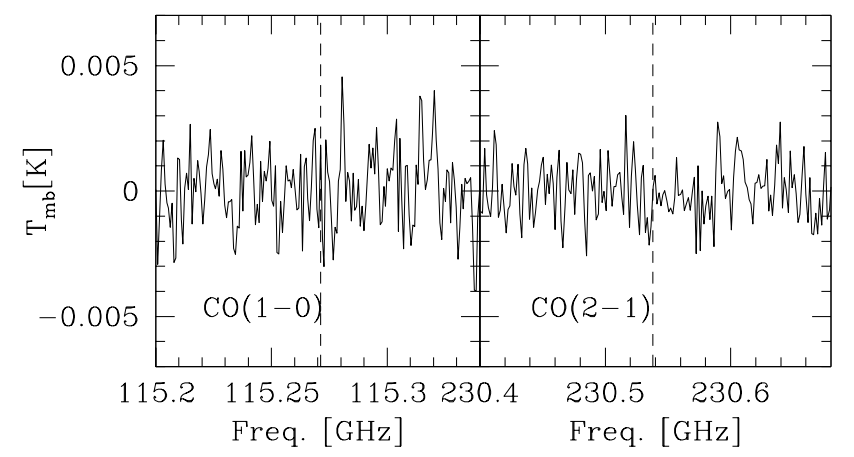

Fig. 8. Stacked spectra obtained by averaging individual spectra of undetected sources after aligning them to the rest frequency of the expected $\mathrm{CO}$ lines. The vertical dashed line indicates the location in the spectra where a faint $\mathrm{CO} J=1-0$ or CO $J=2-1$ line should be detected if some faint emission line were present in individual spectra.

\subsection{Background source count at $24 \mu \mathrm{m}$}

The MIR source catalog of Sharma et al. (2011) contains 912 sources that lie in the sky area covered by the M 33 gaseous disk. They have $24 \mu \mathrm{m}$ fluxes above $0.2 \mathrm{mJy}$, and only 240 sources are brighter than $5 \mathrm{mJy}$. The area of the survey is approximately $0.75 \mathrm{deg}^{2}$, equivalent to $161 \mathrm{kpc}^{2}$ at the distance of M33. At bright flux densities, $F_{24} \geq 5 \mathrm{mJy}$, the background source counts increase at approximately the Euclidean rate, and we expect only 23 sources in the survey area (Papovich et al. 2004), that is, a contamination by background sources lower than $10 \%$. Between 0.2 and $5 \mathrm{mJy}$ the MIR catalog might instead suffer significantly from contamination by background sources because the number density of these increases as the flux decreases. There is evidence that the number of background sources increases at superEuclidean rate below $5 \mathrm{mJy}$ (Papovich et al. 2004). However, the MIR source catalog in the M 33 area is far from being complete below $5 \mathrm{mJy}$ because the sensitivity of the survey varies across the sky, to crowding and diffuse emission in the inner regions of M33. Hence, both MIR sources located in the M33 disk and background sources from more distant sources might not have been detected.

To evaluate the level of background contamination in our sample, we plot in Fig. 9 the radial decline of the number of MIR sources per unit area expressed in $\mathrm{kpc}^{2}$. Owing to the progressive radial decline of star formation per unit area in M 33, we expect that the number density of MIR that are true SF sites declines radially as well. This has been shown to be the case for the whole sample selected by Sharma et al. (2011), although the radial distribution flattens beyond $8 \mathrm{kpc}$. We selected sources with fluxes $F_{24}>5 \mathrm{mJy}$ (bright sample), and with $5>F_{24}>0.2 \mathrm{mJy}$ (faint sample) and plot in Fig. 9 the number of sources per unit area as a function of galactocentric radius. The absence of bright sources beyond $8 \mathrm{kpc}$ (open squares in Fig. 9) does not necessarily imply that star formation stops beyond this radius since SF sites might be fainter at large galactocentric radius. The filled symbols connected by the heavy line in the same figure indicate the radial trend for the faint sample, which contains the 99 sources described in Sect. 2 of this paper. The flatness of the density distribution for fainter sources beyond $8 \mathrm{kpc}$ suggests that this is the likely level of background contamination, although the baryonic surface density also flattens in the outer disk. The apparent weak radial decline of the faint MIR source number density in the outer disk is uncertain because of the deconvolution procedure in the warped region (the tilted rings partially overlap), and we considered the mean value of the observed source density to estimate the background contamination. 


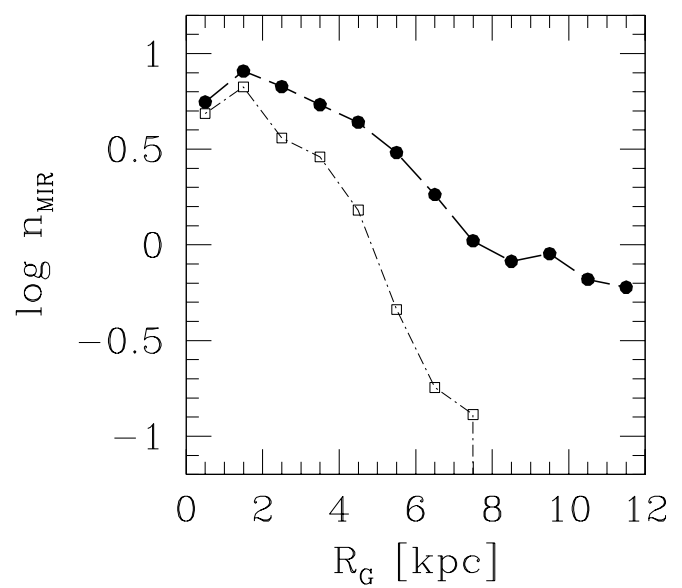

Fig. 9. Number density of MIR sources, $n_{\mathrm{MIR}}$, per kpc ${ }^{2}$ (equivalent to $0.0047 \mathrm{deg}^{2}$ ). The open squares and filled circles show sources in the Sharma et al. (2011) catalog with $24 \mu \mathrm{m}$ flux $F_{24}>5 \mathrm{mJy}$ and $F_{24}<$ $5 \mathrm{mJy}$, respectively.

Figure 9 shows that the number density of sources per $\mathrm{kpc}^{2}$ with $F_{24}<5 \mathrm{mJy}$ and galactocentric distances between 8 and $12 \mathrm{kpc}$ in the MIR catalog varies between 0.9 and 0.6 with a mean value of 0.75 per $\mathrm{kpc}^{2}$. The 99 selected sources lie in an area of approximately $100 \mathrm{kpc}^{2}$ (this is less than what can be inferred by a simple elliptical projection because there is a partial overlap of the projected areas in the warped region), which means that between 60 and 90 of them can be background sources. This estimate is compatible with the higher density inferred from $24 \mu \mathrm{m}$ source counts in fields observed by Spitzer with higher sensitivity. Although our background contamination estimate is an upper limit because some sources might be small SF sites in the outer disk of M 33, the situation is clearly reversed with respect to the bright sample. For the faint sample the majority of the selected sources might be background sources that are unrelated to M33, but are galaxies and quasars at high redshifts (Chary et al. 2004; Papovich et al. 2007). This can explain the non-detection of $\mathrm{CO}$ lines in the selected sources.

The $24 \mu \mathrm{m}$ fluxes for sources in the MIR sample are between 0.4 and $2.8 \mathrm{mJy}$, while for the sample selected by Corbelli et al. (2011), 17 of the 18 SF sites have $24 \mu \mathrm{m}$ fluxes above $3 \mathrm{mJy}$ and have on average a CO $J=2-1$ integrated line brightness of $1 \mathrm{~K} \mathrm{~km} \mathrm{~s}^{-1}$. Although there is no exact linear scaling relation between the brightness of the $\mathrm{CO}$ lines and the $24 \mu \mathrm{m}$ flux, the stacked spectra and the upper limits on the CO line brightness at the location of faint MIR sources presented in this paper are sufficiently low $\left(\leq 0.1 \mathrm{~K} \mathrm{~km} \mathrm{~s}^{-1}\right)$ to suggest that these faint sources might be unrelated to star formation in M 33 . We furthermore underline that none of the selected SF sites of Corbelli et al. (2011) was located beyond $7 \mathrm{kpc}$ and that the two detected sources in the MIR sample presented here have rather weak MIR emission with respect to the rest of the sample, but have associated FUV and $\mathrm{H} \alpha$ emission.

The attempt to select truly SF sites using IRAC colors turns out to be effective for separating MIR sources that are SF sites from variable stars and local galaxies, but the lack of $\mathrm{CO}$ detections around 10 of the 12 observed sources indicates that contamination from faint background sources is still present. The $\mathrm{CO}$ detection in the proximity of two sources of our sample suggests that sources with FUV luminosities higher than $10^{36.8} \mathrm{erg} \mathrm{s}^{-1}$ (if placed at the distance of M33) should be selected to find CO lines, while embedded sources with no UV or optical counterpart are extremely rare because of the short duration of this SF phase (Corbelli et al. 2017). By discarding the 18 sources tagged as galaxies, only 8 of the 81 sources satisfy this selection criteria, and 5 of them lie in the selected area shown in Fig. 3. Two of these (sources s421 and s889) are associated with cataloged GMCs (GMC 325 and GMC 566), and two are sources associated with the newly detected $\mathrm{CO}$ emission presented in this paper (sources s799 and s892). These 4 sources lie within the selected area for IRAC colors and are within the M33 optical radius, at galactocentric distances between 7.5 and $8.1 \mathrm{kpc}$. At a similar distance lies the only source within the selected area with FUV luminosities $>10^{36.8} \mathrm{erg} \mathrm{s}^{-1}$ that has not been observed yet. This is s880, a good candidate for being a SF region with associated $\mathrm{CO}$ emission, although there is no $\mathrm{H} \alpha$ emission in its proximity. Reliable $\mathrm{H} \alpha$ fluxes from compact sources in our database have associated luminosities $\geq 35.8-36 \mathrm{erg} \mathrm{s}^{-1}$, and only sources s421 and s892 of the 81 sources are clearly detected in $\mathrm{H} \alpha$. The paucity of $\mathrm{H} \alpha$ emission can be explained by the IMF incompleteness in low-mass clusters, however, because the Ly-continuum emission decreases much more rapidly than UV emission as the stellar mass in a young cluster decreases.

We underline that in the outer disk of M 33, small SF sites are still present within the optical radius $(8.5 \mathrm{kpc})$, they are rare and can be found by a careful analysis of associated UV emission and IRAC colors, as confirmed by the detection of molecular lines in their close proximity presented in this paper. If our estimate of the background contamination is correct, there might still be only a few other MIR sources in our sample with very faint FUV and CO luminosity that are SF sites in the outer disk of M 33.

\subsection{Evolution of the star-forming region in the southern field}

The total molecular mass recovered by mapping the southern field is about $6 \times 10^{4} M_{\odot}$, as shown in Table 4 and discussed in Sect. 4.1. Most of the molecular gas emission is in the proximity of the isolated HII region in this field, which hosts a stellar cluster whose stellar mass and age have been estimated to be about $6000 M_{\odot}$ and $8 \mathrm{Myr}$ by Sharma et al. (2011). As cataloged by Relaño et al. (2013), the H $\alpha$ emission of MA1 in the southern field is not compact, nor is it distributed in a pure shell (as in the case of RVP87, the HII region in the northern side of the outer disk), but is of mixed morphology. This is indicative of an intermediate age for the SF region associated with it, as confirmed by the spectral energy distribution fits by Sharma et al. (2011) The patchy location of $\mathrm{CO}$ peaks and the high ratio of stellar to molecular gas mass suggests that the molecular cloud is no longer contracting, but is affected by the stellar cluster feedback. The velocity shift between the CO lines and the $21 \mathrm{~cm}$ lines at the same location in the proximity of MA1 is larger than for the detected CO lines in the MIR sample and is consistent with this evolutionary scenario.

The cluster intermediate age is supported by dust properties: dust is still at high temperature and localized rather than being distributed farther away, such as for a more evolved shell-like HII region. The stellar cluster has a low infrared-to-FUV ratio, as found for a few other MIR sources located in the SF disk that have similar $\mathrm{H} \alpha$ morphologies, $24 \mu$ m fluxes, and stellar cluster ages. None of these sources is coincident with massive GMCs, although one is at the boundary of a cataloged cloud (s765) and others lie close to pixels where some $\mathrm{CO}$ emission is present. The sensitivity in the southern field map is higher than that in the all-disk survey for the SF disk of M 33, and it allows recovering weaker $\mathrm{CO}$ lines. In addition, cataloged GMCs have to satisfy a number of requirements concerning the spatial extent and velocity coherence of contiguous pixels, which makes it unlikely 
that molecular gas distributions as patchy as found around MA1 would be identified as one single GMC.

The low TIR/FUV ratio, cluster age, and $\mathrm{H} \alpha$ morphology suggest that in these regions star formation is close to its end and that stellar evolution is reducing the original molecular content while the cluster breaks through the gas. In a lowdensity environment such as the outer disk, the pressure of the hot gas and shock wave is unable to trigger new GMCs and episodes of star formation in the HII region proximity. Hence, molecular hydrogen is fading away as the young stellar cluster evolves.

\section{Disk instabilities and the corotation radius}

We consider the total gaseous disk of M33, made of atomic and molecular gas, which in addition to its self-gravity feels the gravity of the stellar disk. The disk is unstable according to the Toomre criterion out to about $6.5 \mathrm{kpc}$ (Binney \& Tremaine 2008; Corbelli 2003). The ratio of the atomic gas to stellar dispersion in M 33 is about 0.5 , because the gas FWHM is radially constant and equal to about $13 \mathrm{~km} \mathrm{~s}^{-1}$ and the stellar dispersion is about $25 \mathrm{~km} \mathrm{~s}^{-1}$, as observed in the central regions (Corbelli \& Walterbos 2007; Corbelli et al. 2018). Therefore the approximation of the gaseous and stellar disk stability criteria used by Corbelli (2003) and originally proposed by Wang \& Silk (1994) are reliable, as shown by Romeo \& Wiegert (2011). Stellar kinematic maps of galaxies of mass and morphological type similar to M33 in the integral-field spectroscopic survey CALIFA indicate a rather constant radial velocity dispersion (Falcón-Barroso et al. 2017), and this justifies the use of the value measured in the central regions of M 33 throughout the disk. The outermost unstable regions in the disk of M 33 are between 6 and $7 \mathrm{kpc}$, and they coincide with the transition between the stellar and the gas-dominated regime (see Fig. 10 of Corbelli et al. 2014).

\subsection{Perturbation growth across the M33 disk}

We now examine the size of the perturbations with the fastest growth rate using the circular velocities, the gas, and the stellar surface density radial distributions given by Corbelli et al. (2014). The upper panel of Fig. 10 shows the most unstable wavelength according to Jeans criteria and to Toomre criteria as a function of galactocentric radius. The vertical gaseous disk scale height is also shown, and it is computed using the prescription of Elmegreen (2011) for a two-component galaxy disk, stars, and total gas prior to molecular cloud formation.

If perturbations are unstable, they can collapse, and as the density grows, most of the gas will become molecular; the fractional mass of the atomic envelope depends on local conditions and on the size of bound clouds, which is hard to determine because of the limited spatial resolution in M33. The Jeans length gives the smallest size for perturbations that can grow and form molecular clouds, and this should be smaller than the thickness of the gaseous disk. Figure 10 shows that this is indeed the case, and clumps that later condense into molecular clouds can grow out to about $6 \mathrm{kpc}$ (considering the additional gravity provided by the stellar disk) or slightly farther out if the local density is enhanced. We estimated the highest cloud mass as a function of galactocentric distance as a product of the average local gas density times a spherical volume with radius equal to the thickness of the gaseous disk. The bottom panel of Fig. 10 shows this mass and the mass corresponding to the Jeans length. Cloud masses increase radially outward in the inner disk, and the

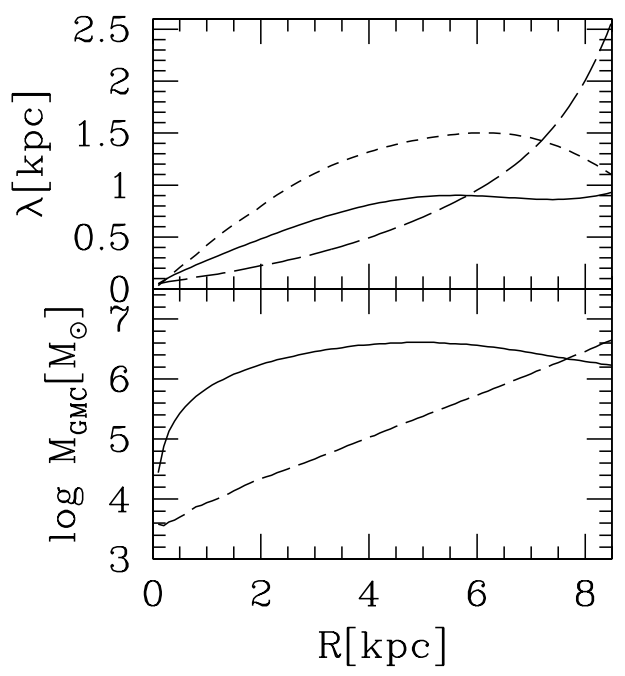

Fig. 10. Unstable perturbations in the M 33 disk. In the upper panel the long- and short-dashed lines show the most unstable wavelength according to Jeans criteria and to Toomre criteria, respectively, as a function of galactocentric radius. The continuous line indicates twice the vertical scale height for the gas. In the bottom panel the predicted molecular cloud masses for perturbations with radius equal to the vertical gaseous disk scale height are shown with a continuous line as a function of galactocentric radius. The long-dashed line indicates the mass corresponding to perturbations with radius equal to one-quarter the Jeans length.

maximum mass value is about $4 \times 10^{6} M_{\odot}$ at $4 \mathrm{kpc}$. Although this mass is in agreement with the highest GMC masses recovered in M33, the radial trend is opposite to what the data in Fig. 1 show at small galactocentric radii. The time for shear to tear a gas condensation apart increases radially outwards and is longer than the free-fall time throughout the SF disk, which means that shear cannot be responsible for the radial decrease of the molecular cloud mass presented in Sect. 2 either.

As shown in Fig. 10, the most unstable wavelength increases radially outward, and this justifies the radial decrease in number density of molecular clouds from the center to the outer disk. The most unstable Toomre length is indicative of the separation between filaments that form in the unstable disk. Therefore the disk instability analysis correctly predicts the observed drop in number density of molecular clouds in the stable outer disk, but it cannot explain the extra growth of individual perturbations in the inner disk where the mean GMC mass is observed to increase toward the center. In the next subsection we examine an additional mechanism, the rotation of the main arm pattern, which can play a role in driving the radial decrease in molecular complex mass in the inner disk. This decrease is not fast, and it is indicative that this mechanism will pile up only a few clouds to provide the extra growth in mass. Only close to the galaxy center is the difference between the observed and the predicted mass (bottom panel of Fig. 10) more evident. However, here the gas vertical scale height is likely underestimated by the model. Furthermore, in the central regions the ISM is highly turbulent, as the shape of the probability distribution function shows (Corbelli et al. 2018), and the high rate of star formation likely triggers the formation of new clouds and enhances their agglomeration into larger complexes by frequent episodes of gas compression.

\subsection{Corotation radius}

In order to derive the speed of the spiral pattern in the disk of M33, we adopted the kinematical method developed by 
Tremaine \& Weinberg (1984, TW). The method was originally devised to provide a model-independent means of measuring the pattern speed of bars in SB0 galaxies. It has subsequently been generalized to non-rigid patterns, that is, with a radially varying pattern speed, by Merrifield et al. (2006), and was used to estimate the pattern speed in nearby galaxies using $\mathrm{HI}$ or $\mathrm{CO}$ emission as mass tracer by Zimmer et al. (2004), for instance. It is assumed that the disk is flat and thin with negligible vertical motions; it is also assumed that the intensity in the adopted tracer, as representative of the surface density of a mass component, obeys a continuity equation along the orbit around the galactic center, meaning that there is no source or sink of the tracer ${ }^{2}$. Both the inclination to the line of sight $i$ and the position angle PA are assumed to be known. Under these hypotheses, the derivation of the pattern speed is quite straightforward. First we produce zeroth- and first-moment images from the $21 \mathrm{~cm}$ and $\mathrm{CO}(2-1)$ cubes and rotate them to align the major axis horizontally ( $x$-axis, positive to the receding SW side). After subtracting the systemic velocity $V_{\mathrm{Hel}}=-180 \mathrm{~km} \mathrm{~s}^{-1}$, for each strip of pixels parallel to the major axis at distance $y$ from it, we evaluate the intensity-weighted line-of-sight mean velocity

$\langle V(y)\rangle=\frac{\int_{-\infty}^{+\infty} I(x, y) v_{\mathrm{LOS}}(x, y) \mathrm{d} x}{\int_{-\infty}^{+\infty} I(x, y) \mathrm{d} x}$,

and similarly, the intensity-weighted mean x-position of the tracer

$\langle x(y)\rangle=\frac{\int_{-\infty}^{+\infty} I(x, y) x \mathrm{~d} x}{\int_{-\infty}^{+\infty} I(x, y) \mathrm{d} x}$.

According to the TW method, the angular speed of the pattern is then given by

$\Omega_{\mathrm{P}}=\frac{1}{\sin i} \frac{\langle V(y)\rangle}{\langle x(y)\rangle}$.

For each strip, at each of the sampled $y$, we will have a pair of $\langle V(y)\rangle,\langle x(y)\rangle$ values that are linearly related in case of a rigid pattern. The slope of the relation $\langle V(y)\rangle$ versus $\langle x(y)\rangle$ is $\Omega_{\mathrm{P}} \times$ $\sin i$.

The results are shown in Figs. 11 and 12. The point distribution is clearly linear and therefore indicative of a welldefined rigid trailing spiral pattern; in addition, the results from the two tracers are remarkably consistent. We obtain the tightest regressions, and best agreement between $\mathrm{HI}$ and $\mathrm{CO}$ results, by adopting a PA $=23^{\circ} \pm 2^{\circ}$, a value quite close to the one derived from the optical images (e.g., 22.7 $7^{\circ}$ in HyperLeda or $23^{\circ}$ in de Vaucouleurs et al. 1991). From the HI we obtain $\Omega_{\mathrm{P}} \times \sin i=18.15 \pm 0.15 \mathrm{~km} \mathrm{~s}^{-1} \mathrm{kpc}^{-1}$, and from the $\operatorname{CO}(2-1)$ we get $18.05 \pm 0.35$ in the same units. The final estimate is then $18.13 \pm 0.14 \mathrm{~km} \mathrm{~s}^{-1} \mathrm{kpc}^{-1}$.

Using the rotation curve and the (inner) disk inclination $\left(i=54^{\circ}\right)$ in Corbelli et al. (2014), we finally obtain a corotation radius $R_{\text {cor }}=4.7 \pm 0.3 \mathrm{kpc}$. The assigned uncertainty includes those on inclination, rotation curve, and $\Omega_{\mathrm{P}} \times \sin i$. Assuming the $21 \mathrm{~cm}$ second moment measures the sound speed (including turbulence), we can locate the sonic point, where the velocity difference between pattern and rotating gas equals the sound speed, at a radius of $3.9 \mathrm{kpc}$. We also performed the preceding analysis

2 In this respect, the components of the ISM do not constitute an ideal tracer, but we can still assume that for the surface density of the component chosen, the relative variations in a parcel of gas are not dramatic within the single orbit.

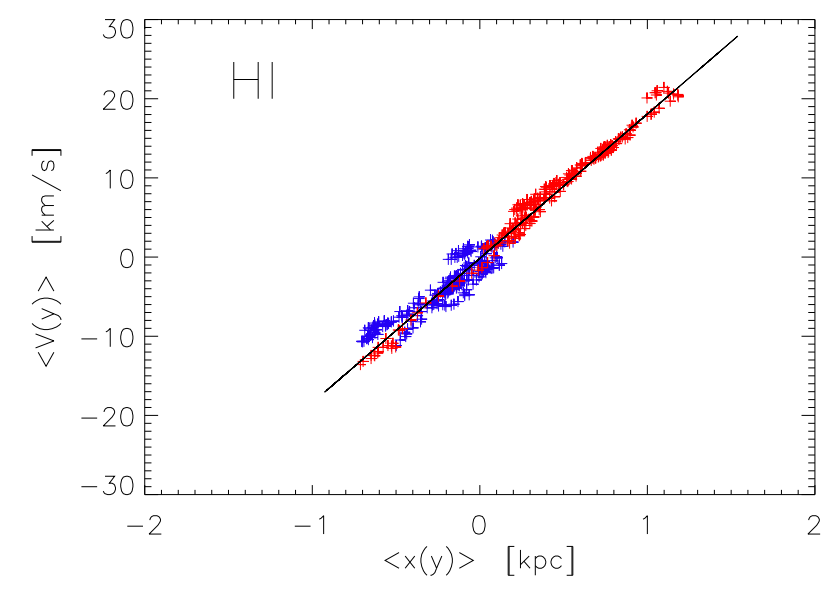

Fig. 11. Intensity-weighted mean line-of-sight HI velocity defined by Eq. (2) vs. the intensity-weighted mean position defined by Eq. (3). Each point represents a different horizontal strip in the moment images, at distance $y$ from the major axis. In red we show data from the far side to the east of the major axis, and in blue we plot data from the near side, to the west of major axis. The solid line is the fitted regression, whose slope gives $\Omega_{\mathrm{P}} \times \sin i$.

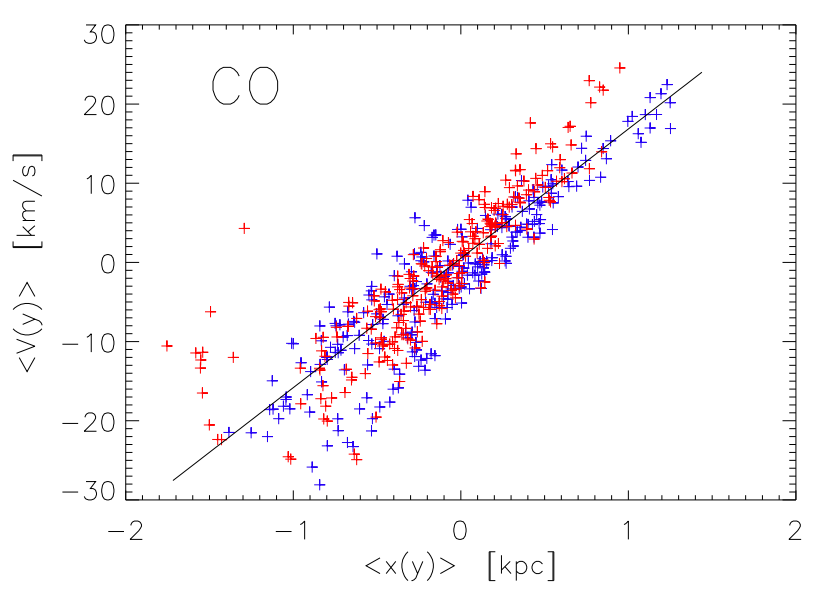

Fig. 12. Same as in Fig. 11, but for the $\mathrm{CO}(2-1)$ moment images.

separately for the two halves of the galaxy, NE and SW of the minor axis. In this case, the corotation is more external in the $\mathrm{NE}, 5.4 \mathrm{kpc}$, than SW, $4.5 \mathrm{kpc}$; we find no significant difference in the mean velocity dispersion.

We summarize the importance of the corotation radius as follows: the lack of molecular clouds in the outer disk as well as the mass distribution of GMCs across the SF disk of M33 is consistent with the mass predicted by the growth of individual condensations in the gaseous unstable disk, except in the innermost regions, where the observed mean GMC mass increases toward the center. When we account for the fast disk rotation with respect to the arm pattern, this discrepancy is alleviated because molecular clouds can experience an extra growth as the arms collect smaller clouds into larger self-shielded complexes inside the corotation radius. The location of the corotation radius is consistent with the galactocentric radius inside which the extra growth of GMC mass is observed.

\section{Summary and conclusions}

We have presented the results of deep observations of CO lines at selected locations in the outer disk of M33 to complement the 
results of existing surveys of molecular clouds in the SF disk and examine possible triggers of molecular cloud mass growth across the disk of the closest blue disk galaxy. We detected CO $J=1-0$ and $J=2-1$ lines near the position of two MIR sources in the outer disk, at about $8 \mathrm{kpc}$ from the center, which have $24 \mu \mathrm{m}$ fluxes of only $0.5 \mathrm{mJy}$ and faint UV and optical counterparts. The CO lines are very narrow, with main-beam peak temperatures of about $0.05-0.1 \mathrm{~K}$. At the location of another ten selected MIR with $24 \mu \mathrm{m}$ fluxes between 0.4 and $2.8 \mathrm{mJy}$ and galactocentric distances between 7.7 and $10.4 \mathrm{kpc}$, we have only upper limits on the $\mathrm{CO}$ line emission that are considerably below the line brightness detected in the proximity of the other two MIR sources.

We also detect CO $J=2-1$ lines in the proximity of MA1, one of the outermost HII regions in the south, at about $7.4 \mathrm{kpc}$ from the center. We mapped the CO $J=2-1$ line emission in an area of about $5 \mathrm{arcmin}^{2}$ and detect $\mathrm{CO}$ lines that imply a total molecular mass of about $6 \times 10^{4} M_{\odot}$. The most prominent line is emitted close to the $\mathrm{H} \alpha$ brightness peak where most of the dust emission is also located. The other CO lines are detected at the boundary of the HII regions: here the gas is likely compressed through shock fronts that develop as the HII region expands, and some cold gas, molecular and atomic, can be found.

It is conceivable that the paucity of CO lines in the outer disk of M33 is enhanced by the lower metal abundance, although the metallicity gradient in the bright disk is not very steep. However, the analysis in this paper has shown that a change in the disk dynamical conditions is likely at the heart of the detected variations of molecular cloud growth across M33. We summarize our main conclusions below.

- The speed of the spiral arm pattern in the inner disk is determined through a kinematical method applied to both HI and $\mathrm{CO}$ data sets. The atomic and molecular gas give consistent results: the arms of $\mathrm{M} 33$ rotate at about $22.4 \mathrm{~km} \mathrm{~s}^{-1} \mathrm{kpc}^{-1}$. This speed is slower than the disk rotation inside the corotation radius, which for M33 is $4.7 \mathrm{kpc}$. Given the HI velocity dispersion, we locate the sonic point at $3.9 \mathrm{kpc}$. This implies that inside $3.9 \mathrm{kpc}$, the arm pattern is able to collect gas condensations into larger clouds and trigger star formation through shocks.

- The Toomre criterion predicts that the disk is stable beyond $6.5 \mathrm{kpc}$ if gravity from stars and gas is considered. Inside $6.5 \mathrm{kpc}$, filaments and gas perturbation can grow. The most unstable wavelength, larger than the Jeans length, increases from the center of the galaxy out to about $6 \mathrm{kpc}$. As the disk vertical thickness is smaller than the most unstable wavelength, the disk vertical scale height sets the maximum mass of molecular complexes, which is rather constant and equal to the measured maximum mass of observed GMCs. In the central region, only low-mass GMCs should be found, but this is not the case.

- The mean molecular mass of cataloged complexes in M33 is instead higher at the galaxy center and decreases radially by a factor 2 out to $4 \mathrm{kpc}$, then it flattens out to about $6.5 \mathrm{kpc}$ and drops beyond this radius. The observed trend for $R<4 \mathrm{kpc}$ can be reconciled with the expected decrease of GMC masses toward the center predicted by disk dynamics when the slow rotation of the arm pattern inside corotation is considered. Arms can collect molecular clouds into larger complexes especially where the disk motion relative to them is supersonic.

- The population of faint MIR sources beyond the SF disk in the sky area where the warped outer disk of M 33 is found is consistent with the density of background source counts at
$24 \mu \mathrm{m}$. This explains the paucity of CO lines detected at the location of selected MIR sources and confirms that SF sites beyond the disk unstable region are rare. Stacking of CO $J=$ $1-0$ and $J=2-1$ spectra for undetected MIR sources has not unveiled any fainter $\mathrm{CO}$ emission. However, narrow $\mathrm{CO}$ lines have been detected at the location of two MIR sources associated with FUV emission even in the outer disk, and molecular cloud masses have been estimated.

- The patchy distribution of detected CO lines in the outer disk region hosting MA1, the local weak MIR-to-FUV ratio, and the morphology of the ionized gas suggest that in MA1 star formation is close to its end, as the HII region expands and dissipates the cold gas. This is is consistent with the age and mass estimate of the YSC hosted by MA1.

Our results imply that internal disk dynamics can then play a crucial role in regulating the growth of gas perturbations that later collapse and form stars across the disk of M33. Star formation sites in the outer disk are not widespread at the present cosmic time. The population of intermediate-mass stars in the far outer disk (Grossi et al. 2011), where no bright FUV knots are found, is likely the result of a past burst of star formation triggered by gas or satellite accretion (Grossi et al. 2008; Mostoghiu et al. 2018). Stellar migration from the inner disk cannot affect regions that are in the far outer disk in less than 1 Gyr (Magrini et al. 2016). CO is found at the location of few MIR sources with FUV emission in the outer disk where the azimuthally averaged $\mathrm{HI}$ column density is still above $1 M_{\odot}$ (Corbelli et al. 2014). This is consistent with the location of young stellar clusters associated with FUV knots in dwarf irregular galaxies (Hunter et al. 2016), where the larger distance and lower metallicity prevent deep local CO searches.

Acknowledgements. We would like to thank the anonymous referee for their comments and careful reading of the original manuscript. E.C. thanks Bruce Elmegreen for stimulating discussion on topics related to this paper. We acknowledge funding from the INAF PRIN-SKA 2017 program 1.05.01.88.0.

\section{References}

Amorín, R., Muñoz-Tuñón, C., Aguerri, J. A. L., \& Planesas, P. 2016, A\&A, 588, A 23

Bigiel, F., Bolatto, A. D., Leroy, A. K., et al. 2010, ApJ, 725, 1159

Binney, J., \& Tremaine, S. 2008, Galactic Dynamics: Second Edition (Princeton University Press)

Braine, J., Ferguson, A. M. N., Bertoldi, F., \& Wilson, C. D. 2007, ApJ, 669, L73

Braine, J., Gratier, P., Contreras, Y., Schuster, K. F., \& Brouillet, N. 2012, A\&A, 548, A52

Braine, J., Rosolowsky, E., Gratier, P., Corbelli, E., \& Schuster, K.-F. 2018, A\&A, 612, A51

Chary, R., Casertano, S., Dickinson, M. E., et al. 2004, ApJS, 154, 80

Colombo, D., Hughes, A., Schinnerer, E., et al. 2014, ApJ, 784, 3

Corbelli, E. 2003, MNRAS, 342, 199

Corbelli, E., \& Walterbos, R. A. M. 2007, ApJ, 669, 315

Corbelli, E., Verley, S., Elmegreen, B. G., \& Giovanardi, C. 2009, A\&A, 495, 479

Corbelli, E., Giovanardi, C., Palla, F., \& Verley, S. 2011, A\&A, 528, A116

Corbelli, E., Thilker, D., Zibetti, S., Giovanardi, C., \& Salucci, P. 2014, A\&A, 572, A23

Corbelli, E., Braine, J., Bandiera, R., et al. 2017, A\&A, 601, A146

Corbelli, E., Elmegreen, B. G., Braine, J., \& Thilker, D. 2018, A\&A, 617, A125

Dekel, A., Zolotov, A., Tweed, D., et al. 2013, MNRAS, 435, 999

de Vaucouleurs, G., de Vaucouleurs, A., Corwin, Jr., H. G., et al. 1991, Third Reference Catalogue of Bright Galaxies. (New York: Springer)

Dib, S., Piau, L., Mohanty, S., \& Braine, J. 2011, MNRAS, 415, 3439

Digel, S., de Geus, E., \& Thaddeus, P. 1994, ApJ, 422, 92

Dobbs, C. L., Pettitt, A. R., Corbelli, E., \& Pringle, J. E. 2018, MNRAS, 478 3793

Druard, C., Braine, J., Schuster, K. F., et al. 2014, A\&A, 567, A118 
Elmegreen, B. G. 2011, ApJ, 737, 10

Elmegreen, D. M., \& Elmegreen, B. G. 1995, ApJ, 445, 591

Falcón-Barroso, J., Lyubenova, M., van de Ven, G., et al. 2017, A\&A, 597, A48

Fitts, A., Boylan-Kolchin, M., Bullock, J. S., et al. 2018, MNRAS, 479, 319

Freedman, W. L., Wilson, C. D., \& Madore, B. F. 1991, ApJ, 372, 455

Freeman, P., Rosolowsky, E., Kruijssen, J. M. D., Bastian, N., \& Adamo, A. 2017, MNRAS, 468, 1769

Fukui, Y., Kawamura, A., Minamidani, T., et al. 2008, ApJS, 178, 56

Gieren, W., Górski, M., Pietrzyński, G., et al. 2013, ApJ, 773, 69

Gil de Paz, A., Boissier, S., Madore, B. F., et al. 2007, ApJS, 173, 185

Gratier, P., Braine, J., Rodriguez-Fernandez, N. J., et al. 2010, A\&A, 512, A68

Gratier, P., Braine, J., Rodriguez-Fernandez, N. J., et al. 2012, A\&A, 542, A108

Gratier, P., Braine, J., Schuster, K., et al. 2017, A\&A, 600, A27

Greenawalt, B. E. 1998, PhD Thesis, New Mexico State University, USA

Grossi, M., Giovanardi, C., Corbelli, E., et al. 2008, A\&A, 487, 161

Grossi, M., Hwang, N., Corbelli, E., et al. 2011, A\&A, 533, A91

Heyer, M., \& Dame, T. M. 2015, ARA\&A, 53, 583

Hoopes, C. G., \& Walterbos, R. A. M. 2000, ApJ, 541, 597

Hunter, D. A., Elmegreen, B. G., \& Gehret, E. 2016, AJ, 151, 136

Kennicutt, Jr., R. C. 1989, ApJ, 344, 685

Kereš, D., Katz, N., Fardal, M., Davé, R., \& Weinberg, D. H. 2009, MNRAS, 395,160

Kobayashi, M. I. N., Inutsuka, S.-I., Kobayashi, H., \& Hasegawa, K. 2017, ApJ, 836, 175

Magrini, L., Corbelli, E., \& Galli, D. 2007, A\&A, 470, 843
Magrini, L., Stanghellini, L., Corbelli, E., Galli, D., \& Villaver, E. 2010, A\&A, 512, A63

Magrini, L., Coccato, L., Stanghellini, L., Casasola, V., \& Galli, D. 2016, A\&A, 588, A91

Mayall, N. U., \& Aller, L. H. 1942, ApJ, 95, 5

Merrifield, M. R., Rand, R. J., \& Meidt, S. E. 2006, MNRAS, 366, L17

Mostoghiu, R., Di Cintio, A., Knebe, A., et al. 2018, MNRAS, 480, 4455

Papovich, C., Dole, H., Egami, E., et al. 2004, ApJS, 154, 70

Papovich, C., Rudnick, G., Le Floc'h, E., et al. 2007, ApJ, 668, 45

Patel, E., Besla, G., \& Sohn, S. T. 2017, MNRAS, 464, 3825

Relaño, M., Verley, S., Pérez, I., et al. 2013, A\&A, 552, A140

Romeo, A. B., \& Wiegert, J. 2011, MNRAS, 416, 1191

Rosolowsky, E. 2005, PASP, 117, 1403

Sharma, S., Corbelli, E., Giovanardi, C., Hunt, L. K., \& Palla, F. 2011, A\&A, 534, A96

Snell, R. L., Carpenter, J. M., \& Heyer, M. H. 2002, ApJ, 578, 229

Thilker, D. A., Hoopes, C. G., Bianchi, L., et al. 2005, ApJ, 619, L67

Tremaine, S., \& Weinberg, M. D. 1984, ApJ, 282, L5

van der Marel, R. P., Fardal, M. A., Sohn, S. T., et al. 2019, ApJ, 872, 24

Verley, S., Hunt, L. K., Corbelli, E., \& Giovanardi, C. 2007, A\&A, 476, 1161

Verley, S., Corbelli, E., Giovanardi, C., \& Hunt, L. K. 2009, A\&A, 493, 453

Wang, B., \& Silk, J. 1994, ApJ, 427, 759

Williams, B. F., Dalcanton, J. J., Dolphin, A. E., Holtzman, J., \& Sarajedini, A. 2009, ApJ, 695, L15

Zimmer, P., Rand, R. J., \& McGraw, J. T. 2004, ApJ, 607, 285 